\title{
ERROR ESTIMATES FOR THE APPROXIMATION OF THE VELOCITY TRACKING PROBLEM WITH BANG-BANG CONTROLS *
}

\author{
Eduardo Casas $^{1}$ and Konstantinos Chrysafinos ${ }^{2}$
}

\begin{abstract}
The velocity tracking problem for the evolutionary Navier-Stokes equations in $2 \mathrm{~d}$ is studied. The controls are of distributed type but the cost functional does not involve the usual quadratic term for the control. As a consequence the resulting controls can be of bang-bang type. First and second order necessary and sufficient conditions are proved. A fully-discrete scheme based on discontinuous (in time) Galerkin approach combined with conforming finite element subspaces in space, is proposed and analyzed. Provided that the time and space discretization parameters, $\tau$ and $h$ respectively, satisfy $\tau \leq C h^{2}$, then $L^{2}$ error estimates are proved for the difference between the states corresponding to locally optimal controls and their discrete approximations.
\end{abstract}

Mathematics Subject Classification. 49J20, 65M60, 49K20, 35K55, 65N30.

Received May 29, 2015. Revised December 22, 2015. Accepted August 2, 2016.

\section{INTRODUCTION}

In this paper, we consider the following problem:

$$
\text { (P) }\left\{\begin{array}{l}
\min J(\mathbf{u}) \\
\mathbf{u} \in \mathcal{U}_{a d}
\end{array}\right.
$$

where

$$
J(\mathbf{u})=\frac{1}{2} \int_{0}^{T} \int_{\Omega}\left|\mathbf{y}_{\mathbf{u}}(x, t)-\mathbf{y}_{d}(x, t)\right|^{2} \mathrm{~d} x \mathrm{~d} t .
$$

Here $\mathbf{y}_{\mathbf{u}}$ denotes the solution of the $2 \mathrm{~d}$ evolution Navier-Stokes equations

$$
\left\{\begin{array}{l}
\mathbf{y}_{t}-\nu \Delta \mathbf{y}+(\mathbf{y} \cdot \nabla) \mathbf{y}+\nabla p=\mathbf{f}+\mathbf{u} \text { in } \Omega_{T}=\Omega \times(0, T) \\
\operatorname{div} \mathbf{y}=0 \text { in } \Omega_{T}, \mathbf{y}(0)=\mathbf{y}_{0} \text { in } \Omega, \mathbf{y}=0 \text { on } \Sigma_{T}=\Gamma \times(0, T),
\end{array}\right.
$$

and $\mathcal{U}_{a d}$ is the set of feasible controls, defined for $-\infty<\alpha_{j}<\beta_{j}<+\infty, j=1,2$, by

$$
\mathcal{U}_{a d}=\left\{\mathbf{u} \in \mathbf{L}^{\infty}\left(\Omega_{T}\right): \alpha_{j} \leq u_{j}(x, t) \leq \beta_{j} \text { a.e. }(x, t) \in \Omega_{T}, j=1,2\right\} .
$$

Keywords and phrases. Evolution Navier-Stokes equations, optimal control, bang-bang controls, a priori error estimates.

* The first author was partially supported by the Spanish Ministerio de Economía y Competitividad under projects MTM201122711 and MTM2014-57531-P.

1 Departmento de Matemática Aplicada y Ciencias de la Computación, E.T.S.I. Industriales y de Telecomunicación, Universidad de Cantabria, Av. Los Castros s/n, 39005 Santander, Spain. eduardo.casas@unican.es

2 Department of Mathematics, School of Applied Mathematics and Physical Sciences, National Technical University of Athens,

Zografou Campus, 15780 Athens, Greece. chrysafinos@math.ntua.gr 
The scope of the above optimal control problem is to match the velocity vector field to a given target field, by influencing the behavior of the system through a control function. The key difference with our previous works [4-6] stems from the absence of the quadratic term for the controls in the cost functional. Hence, despite the fact that the control function is of distributed type and satisfies pointwise constraints, the above formulation can lead to optimal controls of bang-bang type. To better understand the difference between our problem and the standard velocity tracking problem, we recall that in later case the functional is defined by

$$
J_{\lambda}(\mathbf{u})=\frac{1}{2} \int_{0}^{T} \int_{\Omega}\left|\mathbf{y}_{\mathbf{u}}(t, x)-\mathbf{y}_{d}(t, x)\right|^{2} \mathrm{~d} x \mathrm{~d} t+\frac{\lambda}{2} \int_{\Omega}|\mathbf{u}(t, x)|^{2} \mathrm{~d} x \mathrm{~d} t .
$$

Here, the parameter $\lambda>0$ denotes a penalty parameter, which is typically small compared to the actual size of the data, however clearly also acts as a regularization parameter. In addition, the presence of the Tikhonov regularizing term also provides the crucial relation between the control and adjoint variables facilitating the derivation of second order sufficient conditions (see for instance [8]) and hence the derivation of error estimates. To the contrary the absence of the regularizing term leads to loss of regularity and to non-standard second order sufficient condition, and hence to severe technical difficulties both in analysis and in the construction of suitable numerical schemes. Therefore, there are some important mathematical advantages if we include the Tikhonov term in the cost functional, but the goal is to get a velocity field $\overline{\mathbf{y}}$ as close as possible to the desired velocity field $\mathbf{y}_{d}$. In this paper, we study the practical problem without introducing mathematical tricks that can lead to a worst velocity field.

We point out that the second order conditions for bang-bang optimal control problems have been considered recently for pde constrained optimization when semi-linear pdes are involved [3]. For nonlinear evolutionary pdes, to our best knowledge, there are no results apart from the recent works $[8,10]$ concerning the second order analysis (including the possibility of bang-bang controls). For related discussion and references regarding the computational significance of various optimal control problems related to the Navier-Stokes, we refer the reader to $[16]$.

For various results regarding analysis and approximations, including error estimates for the velocity tracking problem for $2 \mathrm{~d}$ Navier-Stokes flows when minimizing functional (1.2), we refer the reader to the recent works of $[4,5]$. The minimization of (1.2) subject to the $3 \mathrm{~d}$ evolutionary Navier-Stokes equations was treated in [6]. The key ingredient of these proofs is the use of suitable second order necessary and sufficient conditions in the spirit of [9] (for the stationary Navier-Stokes) combined with a discontinuous (in time) Galerkin approach for the discretized problem that allows to circumvent the limited regularity of solutions of Navier-Stokes equations. Recall that even for the regularized problem (1.2), the available regularity in the optimal control setting is very limited due to the presence of control constraints, and hence standard techniques developed for the numerical analysis of the uncontrolled Navier-Stokes equations can not be directly applied.

It is clear that in the absence of the regularizing effects due to the quadratic term in the minimizing functional, there are new and severe challenges. On the other hand, the case of bang-bang controls is physically more relevant in a variety of applications and hence the analysis of suitable schemes is very important. Our work is based on two key ingredients. First, we provide a detailed analysis of first and second order optimality conditions, modifying the techniques of [3] to the case of evolutionary Navier-Stokes equations which plays a pivotal role also in derivation of error estimates. In addition, we analyze a numerical scheme based on the discontinuous time-stepping Galerkin scheme for the piecewise constant time combined with standard conforming finite element subspaces for the discretization in space, under the prescribed regularity assumptions imposed by our optimal control problem. To this end we rely on the recent results of [5] where estimates of order $\mathcal{O}\left(h^{2}\right)$ in the $L^{2}\left(0, T ; \mathbf{L}^{2}(\Omega)\right)$ were proved for the error between the state and its fully-discrete approximation, for given controls in $\mathcal{U}_{a d}$. These arguments allows us to rigorously prove strong convergence of the discrete controls, and an estimate of order $o(\sqrt{h})$ for the difference between state and discrete state variables when piecewise constants in space and time are being used for the disrcetization of the controls. Furthermore, this approach can lead to an improved bound of order $\mathcal{O}(h)$ for the states when combined with the variational discretization framework of Hinze [19]. There are two parameters associated to the numerical discretization: $\tau$ and $h$, indicating the size 
of the grids in time and space, respectively. The usual assumption $\tau \leq C h^{2}$ is needed to prove that the discrete equation has a unique solution. The reader should observe that if we discretize the state equation only in time, not in space, then we cannot prove uniqueness of a solution for the resulting elliptic system. Indeed, this discrete elliptic system is very close to the stationary Navier-Stokes system, for which there is no uniqueness result. Therefore, it is not surprising that the discretization parameter $\tau$ is needed to be small compared with $h$ if we want to prove the uniqueness of a solution for the fully discrete system.

For some related earlier work for optimal control problems for the Navier-Stokes equations, we refer the readerto $[1,12,16-18,20,28,30,32]$ and the references cited therein. Related work of discontinuous Galerkin time-stepping approaches within the context of linear, and semi-linear pde constrained optimization problems can be found in $[11,24-26]$.

A few remarks regarding our choice of the discretization scheme follow. The discontinuous in time Galerkin schemes are known to perform well in a variety of problems whose solutions satisfy low regularity properties. The lowest order scheme (in time) considered here, can be viewed as the Implicit Euler scheme. However, a careful inspection of the proof of [5] (see also references within) reveals that the key difference between the analysis of the classical Implicit Euler scheme and its discontinuous (in time) stepping approach is the use of local (in time) approximation tools. As a result, it leads to an efficient analysis of approximation of problems whose solutions satisfy low regularity properties, and in particular to problems where the time-derivative is discontinuous, and hence it is preferable to be discretized in a completely discontinuous fashion. On the other hand, continuous (in time) Galerkin schemes typically require much more regularity than the one anticipated from our optimal control problem.

We close the introduction with a few remarks regarding the three dimensional case. Our results regarding the first and second order optimality conditions remain valid provided that we are dealing with strong solutions of the 3d Navier-Stokes system. In order to guarantee the existence of an optimal control with an associated state which is a strong solution, we have to consider a different cost functional. Similar to [6], we consider the following functional,

$$
\mathcal{J}(\mathbf{y})=\frac{1}{8} \int_{0}^{T}\left\|\mathbf{y}(t)-\mathbf{y}_{d}(t)\right\|_{\mathbf{L}^{4}(\Omega)}^{8} \mathrm{~d} t
$$

which guarantees that the associated state variable to any control belonging to the admissible set, is indeed a strong solution of the 3d Navier-Stokes system. See Remarks 3.12 and 3.14 for additional explanations.

\section{Assumptions And PRELIMINARY RESUlts}

$\Omega$ is a bounded open subset in $\mathbb{R}^{2}$. We assume that its boundary $\Gamma$ is of class $C^{2}$. The outward unit normal vector to $\Gamma$ at a point $x \in \Gamma$ is denoted by $\mathbf{n}(x)$. Given $0<T<+\infty$, we denote $\Omega_{T}=\Omega \times(0, T)$ and $\Sigma_{T}=\Gamma \times(0, T)$. We fix the notation for Sobolev spaces: $\mathbf{H}^{1}(\Omega)=H^{1}\left(\Omega ; \mathbb{R}^{2}\right), \mathbf{H}_{0}^{1}(\Omega)=H_{0}^{1}\left(\Omega ; \mathbb{R}^{2}\right)$, $\mathbf{H}^{-1}(\Omega)=\left(\mathbf{H}_{0}^{1}(\Omega)\right)^{\prime}$ and $\mathbf{W}^{s, p}(\Omega)=W^{s, p}\left(\Omega ; \mathbb{R}^{2}\right)$ for $1 \leq p \leq \infty$ with $s>0$, while $\mathbf{W}_{0}^{s, p}(\Omega)$ denotes the zero trace space of $\mathbf{W}^{s, p}(\Omega)$. We also consider the spaces of integrable functions $\mathbf{L}^{p}(\Omega)=L^{p}\left(\Omega ; \mathbb{R}^{2}\right)$. For a given Banach space $X, L^{p}(0, T ; X)$ will denote the space of measurable functions $f:(0, T) \longrightarrow X$ such that the associated function $t \rightarrow\|f(t)\|_{X}$ belongs to $L^{p}(0, T)$, endowed with the usual norm.

Along this paper, given a number $1 \leq \bar{p} \leq \infty$, and we set

$$
\mathbf{W}_{\bar{p}}^{2,1}\left(\Omega_{T}\right)=\left\{\mathbf{y} \in \mathbf{L}^{\bar{p}}\left(\Omega_{T}\right): \frac{\partial \mathbf{y}}{\partial x_{i}}, \frac{\partial^{2} \mathbf{y}}{\partial x_{i} x_{j}}, \frac{\partial \mathbf{y}}{\partial t} \in \mathbf{L}^{\bar{p}}\left(\Omega_{T}\right), 1 \leq i, j \leq 2\right\} .
$$

equipped with the standard norm. In the Hilbertian case, we follow the usual notation: $\mathbf{H}^{2,1}\left(\Omega_{T}\right)=\mathbf{W}_{2}^{2,1}\left(\Omega_{T}\right)$. We introduce the usual spaces of divergence-free vector fields:

$$
\begin{aligned}
\mathbf{Y}_{\bar{p}} & =\left\{\mathbf{y} \in \mathbf{W}_{0}^{1, \bar{p}}(\Omega): \operatorname{div} \mathbf{y}=\mathbf{0} \text { in } \Omega\right\}, \\
\mathbf{H}_{\bar{p}} & =\left\{\mathbf{y} \in \mathbf{L}^{\bar{p}}(\Omega): \operatorname{div} \mathbf{y}=\mathbf{0} \text { in } \Omega \text { and } \mathbf{y} \cdot \mathbf{n}=0 \text { on } \Gamma\right\} .
\end{aligned}
$$


Finally, we define $\mathbf{W}(0, T)=\left\{\mathbf{y} \in L^{2}\left(0, T ; \mathbf{Y}_{2}\right): \mathbf{y}_{t} \in L^{2}\left(0, T ; \mathbf{Y}_{2}^{\prime}\right)\right\}$. It is well known that $\mathbf{W}(0, T) \subset$ $C_{w}\left([0, T], \mathbf{H}_{2}\right)$, where $C_{w}\left([0, T], \mathbf{H}_{2}\right)$ is the space of weakly continuous functions $\mathbf{y}:[0, T] \longrightarrow \mathbf{H}_{2}$.

To introduce the weak formulation of (1.1) we define the bilinear and trilinear forms $a: \mathbf{H}^{1}(\Omega) \times \mathbf{H}^{1}(\Omega) \longrightarrow \mathbb{R}$ and $c: \mathbf{L}^{4}(\Omega) \times \mathbf{H}^{1}(\Omega) \times \mathbf{H}^{1}(\Omega) \longrightarrow \mathbb{R}$ by

$$
\begin{aligned}
a(\mathbf{y}, \mathbf{z}) & =\nu \int_{\Omega}(\nabla \mathbf{y}: \nabla \mathbf{z}) \mathrm{d} x=\nu \sum_{i, j=1}^{2} \int_{\Omega} \partial_{x_{i}} y_{j} \partial_{x_{i}} z_{j} \mathrm{~d} x \\
c(\mathbf{y}, \mathbf{z}, \mathbf{w}) & =\frac{1}{2}[\hat{c}(\mathbf{y}, \mathbf{z}, \mathbf{w})-\hat{c}(\mathbf{y}, \mathbf{w}, \mathbf{z})] \text { with } \hat{c}(\mathbf{y}, \mathbf{z}, \mathbf{w})=\sum_{i, j=1}^{2} \int_{\Omega} \mathbf{y}_{j}\left(\frac{\partial \mathbf{z}_{i}}{\partial x_{j}}\right) \mathbf{w}_{i} \mathrm{~d} x .
\end{aligned}
$$

Now, we seek $\mathbf{y} \in \mathbf{W}(0, T)$ such that for a.e. $t \in(0, T)$,

$$
\left\{\begin{array}{l}
\left(\mathbf{y}_{t}, \mathbf{w}\right)+a(\mathbf{y}, \mathbf{w})+c(\mathbf{y}, \mathbf{y}, \mathbf{w})=(\mathbf{f}+\mathbf{u}, \mathbf{w}) \quad \forall \mathbf{w} \in \mathbf{Y}_{2} \\
\mathbf{y}(0)=\mathbf{y}_{0}
\end{array}\right.
$$

Above $(\cdot, \cdot)$ denotes the scalar product in $\mathbf{L}^{2}(\Omega)$. This notation will be frequently used along the paper and $\|\cdot\|$ will denote the associated norm. Any other norm will be indicated by a subscript.

The following hypotheses will be assumed along this paper

(H1) The data of (1.1) satify: $\nu>0, \mathbf{f} \in \mathbf{L}^{\bar{p}}\left(\Omega_{T}\right)$ and $\mathbf{y}_{0} \in \mathbf{W}^{2-\frac{2}{p}, \bar{p}}(\Omega) \cap \mathbf{Y}_{2}$. We will assume that $3<\bar{p}<+\infty$ unless something different is indicated.

(H2) The data in the cost functional $J$ fulfills: $\mathbf{y}_{d} \in \mathbf{L}^{4}\left(\Omega_{T}\right) \cap \mathbf{L}^{\bar{p}}\left(\Omega_{T}\right)$.

The following theorem analyzes the state equation.

Theorem 2.1. For every $\mathbf{u} \in \mathbf{L}^{\bar{p}}\left(\Omega_{T}\right)$ the state equation (1.1) has a unique solution $\mathbf{y}_{\mathbf{u}} \in \mathbf{W}_{\bar{p}}^{2,1}\left(\Omega_{T}\right)$ and an associate pressure $p_{\mathbf{u}} \in L^{\bar{p}}\left(0, T ; W^{1, \bar{p}}(\Omega)\right)$, which is unique up to the addition of a function of $L^{\bar{p}}(0, T)$. Moreover, the following estimate holds

$$
\left\|\mathbf{y}_{\mathbf{u}}\right\|_{\mathbf{W}_{\bar{p}}^{2,1}\left(\Omega_{T}\right)}+\left\|\nabla p_{\mathbf{u}}\right\|_{\mathbf{L}^{\bar{p}}\left(\Omega_{T}\right)} \leq C_{\mathbf{u}}\left(\|\mathbf{f}+\mathbf{u}\|_{\mathbf{L}^{\bar{p}}\left(\Omega_{T}\right)}+\left\|\mathbf{y}_{0}\right\|_{\mathbf{W}^{2-\frac{2}{\bar{p}}, \bar{p}}(\Omega)}\right),
$$

where $C_{\mathbf{u}}$ depends on $\|\mathbf{f}+\mathbf{u}\|_{\mathbf{L}^{2}\left(\Omega_{T}\right)}$ and $\left\|\mathbf{y}_{0}\right\|_{\mathbf{Y}_{2}}$. Furthermore, the constant $C_{\mathbf{u}}$ in (2.2) can be chosen the same for every $\mathbf{u} \in \mathcal{U}_{a d}$.

Proof. Since $n=2$, it is well-known that (1.1) has a unique solution $\mathbf{y}_{\mathbf{u}} \in \mathbf{W}(0, T)$, and there exists a distribution $p_{\mathbf{u}} \in \mathcal{D}\left(\Omega_{T}\right)$ unique up to the addition of a distribtuion of $\mathcal{D}(0, T)$. Thanks to the $C^{2}$ regularity of $\Gamma$, additional regularity is proved for $(\mathbf{y}, p)$. Indeed, we have that $\mathbf{y}_{\mathbf{u}} \in \mathbf{H}^{2,1}\left(\Omega_{T}\right) \cap C\left([0, T], \mathbf{Y}_{2}\right)$ and $p_{\mathbf{u}} \in L^{2}\left(0, T ; H^{1}(\Omega)\right)$; see, for instance, $[21,23,31]$. Furthermore, there exists a constant $C_{0}$ depending on $\|\mathbf{f}+\mathbf{u}\|_{\mathbf{L}^{2}\left(\Omega_{T}\right)}$ and $\left\|\mathbf{y}_{0}\right\|_{\mathbf{Y}_{2}}$ such that

$$
\left\|\mathbf{y}_{\mathbf{u}}\right\|_{\mathbf{H}^{2,1}\left(\Omega_{T}\right)}+\left\|\mathbf{y}_{\mathbf{u}}\right\|_{L^{\infty}\left(0, T ; \mathbf{Y}_{2}\right)} \leq C_{0} .
$$

The boundedness of $\mathcal{U}_{a d}$ in $\mathbf{L}^{\infty}\left(\Omega_{T}\right)$ implies that $C_{0}$ can be chosen the same for all $\mathbf{u} \in \mathcal{U}_{\text {ad }}$. Since $\Omega \subset \mathbb{R}^{2}$, then we have the continuous embedding $\mathbf{Y}_{2} \subset \mathbf{L}^{\bar{p}}(\Omega)$. Using this fact and the above inequality we deduce

$$
\left\|\mathbf{y}_{\mathbf{u}}\right\|_{\mathbf{L}^{\bar{p}}\left(\Omega_{T}\right)} \leq C_{1}\left\|\mathbf{y}_{\mathbf{u}}\right\|_{L^{\infty}\left(0, T ; \mathbf{Y}_{2}\right)} \leq C_{1} C_{0}
$$

for some constant $C_{1}$ depending only on $\Omega$ and $\bar{p}$.

Finally, the estimate (2.2) follows from the result by Solonnikov ([29], Thm. 4.2) with $a=0$ and $a_{j}=y_{j}$, $j=1,2$, and $\mathbf{y}_{\mathbf{u}}=\left(y_{1}, y_{2}\right)$. 
Corollary 2.2. Assume that $\bar{p}>3$. Then, there exists a constant $M_{\boldsymbol{\alpha}, \boldsymbol{\beta}}$ such that $\forall \mathbf{u} \in \mathcal{U}_{a d}$

$$
\left\|\mathbf{y}_{\mathbf{u}}\right\|_{C\left([0, T] ; \mathbf{Y}_{\bar{p}}\right)}+\left\|\mathbf{y}_{\mathbf{u}}\right\|_{\mathbf{C}^{0,1-\frac{3}{p}}\left(\bar{\Omega}_{T}\right)} \leq M_{\boldsymbol{\alpha}, \boldsymbol{\beta}}\left(\|\mathbf{f}+\mathbf{u}\|_{\mathbf{L}^{\bar{p}}\left(\Omega_{T}\right)}+\left\|\mathbf{y}_{0}\right\|_{\mathbf{W}^{2-\frac{2}{\bar{p}}, \bar{p}}(\Omega)}\right),
$$

where $\mathbf{C}^{0,1-\frac{3}{p}}\left(\bar{\Omega}_{T}\right)$ is the space of Hölder functions in $\bar{\Omega}_{T}$ of order $1-\frac{3}{\bar{p}}$.

Proof. The estimate in the space $C\left([0, T] ; \mathbf{Y}_{\bar{p}}\right)$ follows from $(2.2)$ and the fact that $\mathbf{W}_{\bar{p}}^{2,1}\left(\Omega_{T}\right) \subset C\left([0, T] ; \mathbf{Y}_{\bar{p}}\right)$, with continuous embedding; (see [29], Thm. 2.1). The Hölder estimate is a consequence of (2.2) and the Sobolev inclusion $\mathbf{W}^{1, \bar{p}}\left(\Omega_{T}\right) \subset \mathbf{C}^{0,1-\frac{3}{\bar{p}}}\left(\bar{\Omega}_{T}\right)$ along with the obvious embedding $\mathbf{W}_{\bar{p}}^{2,1}\left(\Omega_{T}\right) \subset \mathbf{W}^{1, \bar{p}}\left(\Omega_{T}\right)$.

Remark 2.3. Since the definition of $\mathcal{U}_{a d}$ is exclusively related to the parameters $\boldsymbol{\alpha}$ and $\boldsymbol{\beta}$, the notation $M_{\boldsymbol{\alpha}, \boldsymbol{\beta}}$ only tries to emphasize this dependence.

Corollary 2.4. Assume that $\bar{p}>3$. Let $\left\{\mathbf{u}_{k}\right\}_{k=1}^{\infty} \subset \mathbf{L}^{\bar{p}}\left(\Omega_{T}\right)$ be a sequence converging weakly to $\mathbf{u}$ in $\mathbf{L}^{\bar{p}}\left(\Omega_{T}\right)$. Then, the following strong convergence holds: $\mathbf{y}_{\mathbf{u}_{k}} \rightarrow \mathbf{y}_{\mathbf{u}}$ in $\mathbf{C}\left(\bar{\Omega}_{T}\right) \cap \mathbf{L}^{\bar{p}}\left(0, T ; \mathbf{W}_{0}^{1, \bar{p}}(\Omega)\right)$.

Proof. This is an immediate consequence of $(2.2),(2.3)$, and the compactness of the inclusions $\mathbf{W}_{\bar{p}}^{2,1}\left(\Omega_{T}\right) \subset$ $\mathbf{L}^{\bar{p}}\left(0, T ; \mathbf{W}_{0}^{1, \bar{p}}(\Omega)\right)$ and $\mathbf{C}^{0,1-\frac{3}{p}}\left(\bar{\Omega}_{T}\right) \subset \mathbf{C}\left(\bar{\Omega}_{T}\right)$.

We finish this section analyzing the mapping $G: \mathbf{L}^{\bar{p}}\left(\Omega_{T}\right) \rightarrow \mathbf{W}_{\bar{p}}^{2,1}\left(\Omega_{T}\right) \cap C\left([0, T] ; \mathbf{Y}_{\bar{p}}\right)$ that associates to each control $\mathbf{u}$ the corresponding state $G(\mathbf{u})=\mathbf{y}_{\mathbf{u}}$, through (2.1). The next theorem was proved in $[2,6]$ for $\bar{p}=2$.

Theorem 2.5. Let $2 \leq \bar{p}<+\infty$. Then, the mapping $G$ is of class $C^{\infty}$. Moreover, for any $\mathbf{u}, \mathbf{v}, \mathbf{v}_{i} \in \mathbf{L}^{\bar{p}}\left(\Omega_{T}\right)$, $i=1,2$, if we denote $\mathbf{y}_{\mathbf{u}}=G(\mathbf{u}), \mathbf{z}_{\mathbf{v}}=G^{\prime}(\mathbf{u}) \mathbf{v}, \mathbf{z}_{\mathbf{v}_{i}}=G^{\prime}(\mathbf{u}) \mathbf{v}_{i}$, and $\mathbf{z}_{\mathbf{v}_{1} \mathbf{v}_{2}}=G^{\prime \prime}(\mathbf{u})\left(\mathbf{v}_{1}, \mathbf{v}_{2}\right)$, then $\mathbf{z}_{\mathbf{v}}$ and $\mathbf{z}_{\mathbf{v}_{1} \mathbf{v}_{2}}$ are the unique solutions of the following equations

$$
\begin{gathered}
\left\{\begin{array}{l}
\frac{\partial \mathbf{z}_{\mathbf{v}}}{\partial t}-\nu \Delta \mathbf{z}_{\mathbf{v}}+\left(\mathbf{y}_{\mathbf{u}} \cdot \nabla\right) \mathbf{z}_{\mathbf{v}}+\left(\mathbf{z}_{\mathbf{v}} \cdot \nabla\right) \mathbf{y}_{\mathbf{u}}+\nabla r_{\mathbf{v}}=\mathbf{v} \text { in } \Omega_{T}, \\
\operatorname{div} \mathbf{z}_{\mathbf{v}}=0 \text { in } \Omega_{T}, \mathbf{z}_{\mathbf{v}}(0)=0 \text { in } \Omega, \mathbf{z}_{\mathbf{v}}=0 \text { on } \Sigma_{T},
\end{array}\right. \\
\left\{\begin{array}{c}
\frac{\partial \mathbf{z}_{\mathbf{v}_{1} \mathbf{v}_{2}}}{\partial t}-\nu \Delta \mathbf{z}_{\mathbf{v}_{1} \mathbf{v}_{2}}+\left(\mathbf{y}_{\mathbf{u}} \cdot \nabla\right) \mathbf{z}_{\mathbf{v}_{1} \mathbf{v}_{2}}+\left(\mathbf{z}_{\mathbf{v}_{1} \mathbf{v}_{2}} \cdot \nabla\right) \mathbf{y}_{\mathbf{u}} \\
\quad+\left(\mathbf{z}_{\mathbf{v}_{2}} \cdot \nabla\right) \mathbf{z}_{\mathbf{v}_{1}}+\left(\mathbf{z}_{\mathbf{v}_{1}} \cdot \nabla\right) \mathbf{z}_{\mathbf{v}_{2}}+\nabla r_{\mathbf{v}_{1} \mathbf{v}_{2}}=0 \text { in } \Omega_{T}, \\
\operatorname{div} \mathbf{z}_{\mathbf{v}_{1} \mathbf{v}_{2}}=0 \text { in } \Omega_{T}, \mathbf{z}_{\mathbf{v}_{1} \mathbf{v}_{2}}(0)=0 \text { in } \Omega, \mathbf{z}_{\mathbf{v}_{1} \mathbf{v}_{2}}=0 \text { on } \Sigma_{T},
\end{array}\right.
\end{gathered}
$$

for some $r_{\mathbf{v}}, r_{\mathbf{v}_{1} \mathbf{v}_{2}} \in L^{\bar{p}}\left(0, T ; W^{1, \bar{p}}(\Omega)\right)$, which are unique up to the addition of a function of $L^{2}(0, T)$.

Proof. We define the mapping

$$
\begin{aligned}
& \mathcal{F}:\left(\mathbf{W}_{\bar{p}}^{2,1}\left(\Omega_{T}\right) \cap \mathbf{C}\left([0, T] ; \mathbf{Y}_{\bar{p}}\right)\right) \times \mathbf{L}^{\bar{p}}\left(\Omega_{T}\right) \longrightarrow \mathbf{L}^{\bar{p}}\left(0, T ; \mathbf{H}_{\bar{p}}\right) \times\left(\mathbf{W}^{2-\frac{2}{\bar{p}}, \bar{p}}(\Omega) \cap \mathbf{Y}_{\bar{p}}\right) \\
& \mathcal{F}(\mathbf{y}, \mathbf{u})=\left(\frac{\partial \mathbf{y}}{\partial t}+\mathbf{P}_{\mathbf{H}}[-\nu \Delta \mathbf{y}+(\mathbf{y} \cdot \nabla) \mathbf{y}-(\mathbf{f}+\mathbf{u})], \mathbf{y}(0)-\mathbf{y}_{0}\right),
\end{aligned}
$$

where $\mathbf{P}_{\mathbf{H}}: \mathbf{L}^{2}\left(\Omega_{T}\right) \longrightarrow \mathbf{L}^{2}\left(0, T ; \mathbf{H}_{2}\right)$ denotes the Leray projection operator. Since $\Gamma$ is $C^{2}$, we have that $\mathbf{P}_{\mathbf{H}} \mathbf{u} \in \mathbf{L}^{\bar{p}}\left(0, T ; \mathbf{H}_{\bar{p}}\right)$ for every $\mathbf{u} \in \mathbf{L}^{\bar{p}}\left(\Omega_{T}\right)$, and $\mathbf{P}_{\mathbf{H}}$ is continuous from $\mathbf{L}^{\bar{p}}\left(\Omega_{T}\right)$ to $\mathbf{L}^{\bar{p}}\left(0, T ; \mathbf{H}_{\bar{p}}\right)$. This can be easily proved by using the construction of the projection $\mathbf{P}_{\mathbf{H}} \mathbf{u}$; see, for instance ([31], Thm. 1.1.5 and Rem. 1.1.6).

Let us check that $\mathcal{F}$ is well-defined. Given $\mathbf{y} \in \mathbf{W}_{\bar{p}}^{2,1}\left(\Omega_{T}\right)$, we have that $\Delta \mathbf{y}, \nabla \mathbf{y} \in \mathbf{L}^{\bar{p}}\left(\Omega_{T}\right)$. Moreover, with (2.3) we deduce that $(\mathbf{y} \cdot \nabla) \mathbf{y} \in \mathbf{L}^{\bar{p}}\left(\Omega_{T}\right)$. Hence, the first component of $\mathcal{F}(\mathbf{y}, \mathbf{u})$ belongs to $\mathbf{L}^{\bar{p}}\left(0, T ; \mathbf{H}_{\bar{p}}\right)$. For the second component, it is enough to use that the mapping $\mathbf{y} \in \mathbf{W}_{\bar{p}}^{2,1}\left(\Omega_{T}\right) \longrightarrow \mathbf{y}(0) \in \mathbf{W}^{2-\frac{2}{\bar{p}}, \bar{p}}(\Omega)$ is linear 
and continuous; (see [22], Lem. 3.4; p. 82). Note also that since $\mathbf{y} \in \mathbf{W}_{\bar{p}}^{2,1}\left(\Omega_{T}\right) \cap C\left([0, T] ; \mathbf{Y}_{\bar{p}}\right)$, we also have that $\mathbf{y}(0) \in \mathbf{Y}_{\bar{p}}$. It is obvious that $\mathcal{F}$ is of class $C^{\infty}$ and

$$
\frac{\partial \mathcal{F}}{\partial \mathbf{y}}(\mathbf{y}, \mathbf{u}) \mathbf{z}=\left(\frac{\partial \mathbf{z}}{\partial t}+\mathbf{P}_{\mathbf{H}}[-\nu \Delta \mathbf{z}+(\mathbf{y} \cdot \nabla) \mathbf{z}+(\mathbf{z} \cdot \nabla) \mathbf{y}], \mathbf{z}(0)\right) .
$$

Now, we observe that

$$
\begin{aligned}
\left(\mathbf{P}_{\mathbf{H}}[-\nu \Delta \mathbf{z}+(\mathbf{y} \cdot \nabla) \mathbf{z}+(\mathbf{z} \cdot \nabla) \mathbf{y}], \boldsymbol{\psi}\right) & =(-\nu \Delta \mathbf{z}+(\mathbf{y} \cdot \nabla) \mathbf{z}+(\mathbf{z} \cdot \nabla) \mathbf{y}, \boldsymbol{\psi}) \\
& =a(\mathbf{z}, \boldsymbol{\psi})+c(\mathbf{y}, \mathbf{z}, \boldsymbol{\psi})+c(\mathbf{z}, \mathbf{y}, \boldsymbol{\psi}) \quad \forall \boldsymbol{\psi} \in \mathbf{Y}_{2} .
\end{aligned}
$$

Therefore, $\frac{\partial \mathcal{F}}{\partial \mathbf{y}}(\mathbf{y}, \mathbf{u}) \mathbf{z}=\left(\mathbf{v}, \mathbf{z}_{0}\right)$, with $\left(\mathbf{v}, \mathbf{z}_{0}\right) \in L^{2}\left(0, T ; \mathbf{H}_{2}\right) \times \mathbf{Y}_{2}$, if and only if

$$
\left\{\begin{array}{l}
\left(\mathbf{z}_{t}, \boldsymbol{\psi}\right)+a(\mathbf{z}, \boldsymbol{\psi})+c(\mathbf{z}, \mathbf{y}, \boldsymbol{\psi})+c(\mathbf{y}, \mathbf{z}, \boldsymbol{\psi})=(\mathbf{v}, \boldsymbol{\psi}) \forall \boldsymbol{\psi} \in \mathbf{Y}_{2} \\
\mathbf{z}(0)=\mathbf{z}_{0}
\end{array}\right.
$$

or equivalently

$$
\left\{\begin{array}{l}
\frac{\partial \mathbf{z}}{\partial t}-\nu \Delta \mathbf{z}+(\mathbf{y} \cdot \nabla) \mathbf{z}+(\mathbf{z} \cdot \nabla) \mathbf{y}+\nabla r_{\mathbf{v}}=\mathbf{v} \text { in } \Omega_{T}, \\
\operatorname{div} \mathbf{z}=0 \text { in } \Omega_{T}, \mathbf{z}(0)=\mathbf{z}_{0} \text { in } \Omega, \mathbf{z}=0 \text { on } \Sigma_{T} .
\end{array}\right.
$$

Using again ([29], Thm. 4.2), we deduce that the above equation has unique solution $\mathbf{z}_{\mathbf{v}} \in \mathbf{W}_{\bar{p}}^{2,1}\left(\Omega_{T}\right) \cap$ $C\left([0, T] ; \mathbf{Y}_{\bar{p}}\right)$ for every $\left(\mathbf{v}, \mathbf{z}_{0}\right) \in \mathbf{L}^{\bar{p}}\left(0, T ; \mathbf{H}_{\bar{p}}\right) \times \mathbf{W}^{2-\frac{2}{\bar{p}}, \bar{p}}(\Omega) \cap \mathbf{Y}_{\bar{p}}$, and the relation $\left(\mathbf{v}, \mathbf{z}_{0}\right) \rightarrow \mathbf{z}$ is continuous. Hence, $\frac{\partial \mathcal{F}}{\partial \mathbf{y}}(\mathbf{y}, \mathbf{u}): \mathbf{W}_{\bar{p}}^{2,1}\left(\Omega_{T}\right) \times \mathbf{L}^{\bar{p}}\left(\Omega_{T}\right) \longrightarrow \mathbf{L}^{\bar{p}}\left(0, T ; \mathbf{H}_{\bar{p}}\right) \times \mathbf{W}^{2-\frac{2}{\bar{p}}, \bar{p}}(\Omega) \cap \mathbf{Y}_{\bar{p}}$ is an isomorphism. Therefore, we can apply the implicit function theorem to the equation $\mathcal{F}(G(\mathbf{u}), \mathbf{u})=0$ to deduce that $G$ is of class $C^{\infty}$. Moreover, (2.4) and (2.5) follow easily from the definition of $\mathcal{F}$.

Corollary 2.6. There exist constants $L_{\infty}$ and $L_{2}$ such that $\forall \mathbf{u}_{1}, \mathbf{u}_{2} \in \mathcal{U}_{a d}$

$$
\begin{aligned}
&\left\|\mathbf{y}_{\mathbf{u}_{2}}-\mathbf{y}_{\mathbf{u}_{1}}\right\|_{\mathbf{L}^{\infty}\left(\Omega_{T}\right)} \leq L_{\infty}\left\|\mathbf{u}_{2}-\mathbf{u}_{1}\right\|_{\mathbf{L}^{2}\left(\Omega_{T}\right)}^{\frac{2}{\bar{p}}}, \\
&\left\|\mathbf{y}_{\mathbf{u}_{2}}-\mathbf{y}_{\mathbf{u}_{1}}\right\|_{\mathbf{L}^{2}\left(\Omega_{T}\right)} \leq L_{2}\left\|\mathbf{u}_{2}-\mathbf{u}_{1}\right\|_{\mathbf{L}^{2}\left(\Omega_{T}\right)},
\end{aligned}
$$

where $\bar{p}>3$.

Proof. From Theorem 2.5, (2.3), and ([29], Thm. 4.2) we have

$$
\left\|\mathbf{y}_{\mathbf{u}_{2}}-\mathbf{y}_{\mathbf{u}_{1}}\right\|_{\mathbf{L}^{\infty}\left(\Omega_{T}\right)} \leq \max _{0 \leq \rho \leq 1}\left\|G^{\prime}\left(\mathbf{u}_{1}+\rho\left(\mathbf{u}_{2}-\mathbf{u}_{1}\right)\right)\right\|\left\|\mathbf{u}_{2}-\mathbf{u}_{1}\right\|_{\mathbf{L}^{\bar{p}}\left(\Omega_{T}\right)} \leq C\left\|\mathbf{u}_{2}-\mathbf{u}_{1}\right\|_{\mathbf{L}^{\bar{p}}\left(\Omega_{T}\right)},
$$

where $C$ can be taken independently of $\mathbf{u}_{1}, \mathbf{u}_{2} \in \mathcal{U}_{a d}$. Now, using the boundedness of $\mathcal{U}_{a d}$ in $\mathbf{L}^{\infty}\left(\Omega_{T}\right)$ we infer (2.6) from the above inequality. The estimate (2.7) is well known.

\section{AnAlysis of the CONTROL PROBlem}

In this section, we establish the necessary and sufficient optimality conditions for a local minimum of $(\mathrm{P})$. We distinguish between weak and strong local minima. Let us give the precise meaning of these notions.

Definition 3.1. We say that a control $\overline{\mathbf{u}} \in \mathcal{U}_{a d}$ is a local minimum of $(\mathrm{P})$ in the $\mathbf{L}^{p}\left(\Omega_{T}\right)$ sense, $1 \leq p \leq \infty$, if there exists $\varepsilon>0$ such that $J(\overline{\mathbf{u}}) \leq J(\mathbf{u})$ for all $\mathbf{u} \in \mathcal{U}_{a d} \cap B_{\varepsilon}(\overline{\mathbf{u}})$, where $B_{\varepsilon}(\overline{\mathbf{u}})$ is the ball of $\mathbf{L}^{p}\left(\Omega_{T}\right)$ centered at $\overline{\mathbf{u}}$ with radius $\varepsilon$. We say that $\overline{\mathbf{u}}$ is a strict local minimum if the previous inequality is strict for every $\mathbf{u} \neq \overline{\mathbf{u}}$.

Since $\mathcal{U}_{a d}$ is bounded in $\mathbf{L}^{\infty}\left(\Omega_{T}\right)$, it is immediate to check that $\overline{\mathbf{u}}$ is a local minimum in the $\mathbf{L}^{p}\left(\Omega_{T}\right)$ sense with $p<\infty$ if an only if it is a local minimum in the $\mathbf{L}^{1}\left(\Omega_{T}\right)$ sense. In addition, if $\overline{\mathbf{u}}$ is a local minimum in the $\mathbf{L}^{\infty}\left(\Omega_{T}\right)$ sense, then it is a local minimum in the $\mathbf{L}^{p}\left(\Omega_{T}\right)$ sense for every $1 \leq p<\infty$. The contrary is not necessarily true. In the sequel, whenever we say that $\overline{\mathbf{u}}$ is a local minimum of $(\mathrm{P})$, it should be intended in the $\mathbf{L}^{2}\left(\Omega_{T}\right)$ sense. 
We start the analysis of the control problem by proving the differentiability of the cost functional.

Theorem 3.2. Let $2 \leq \bar{p}<+\infty$. Then, the cost functional $J: \mathbf{L}^{\bar{p}}\left(\Omega_{T}\right) \longrightarrow \mathbb{R}$ is of class $C^{\infty}$ and for every $\mathbf{u}, \mathbf{v} \in \mathbf{L}^{\bar{p}}\left(\Omega_{T}\right)$ we have

$$
\begin{aligned}
J^{\prime}(\mathbf{u}) \mathbf{v} & =\int_{0}^{T} \int_{\Omega} \varphi_{\mathbf{u}} \mathbf{v} \mathrm{d} x \mathrm{~d} t \\
J^{\prime \prime}(\mathbf{u}) \mathbf{v}^{2} & =\int_{0}^{T} \int_{\Omega}\left(\left|\mathbf{z}_{\mathbf{v}}\right|^{2}-2\left(\mathbf{z}_{\mathbf{v}} \cdot \nabla\right) \mathbf{z}_{\mathbf{v}} \varphi_{\mathbf{u}}\right) \mathrm{d} x \mathrm{~d} t,
\end{aligned}
$$

where $\mathbf{z}_{\mathbf{v}}=G^{\prime}(\mathbf{u}) \mathbf{v}$ is the solution of $(2.4)$ and $\boldsymbol{\varphi}_{\mathbf{u}} \in \mathbf{W}_{\bar{p}}^{2,1}\left(\Omega_{T}\right) \cap C\left([0, T] ; \mathbf{Y}_{\bar{p}}\right)$ is the unique element satisfying for every $\mathbf{w} \in \mathbf{Y}_{2}$

$$
\left\{\begin{array}{l}
-\left(\boldsymbol{\varphi}_{\mathbf{u}, t}, \mathbf{w}\right)+a\left(\boldsymbol{\varphi}_{\mathbf{u}}, \mathbf{w}\right)+c\left(\mathbf{w}, \mathbf{y}_{\mathbf{u}}, \boldsymbol{\varphi}_{\mathbf{u}}\right)+c\left(\mathbf{y}_{\mathbf{u}}, \mathbf{w}, \boldsymbol{\varphi}_{\mathbf{u}}\right)=\left(\mathbf{y}_{\mathbf{u}}-\mathbf{y}_{d}, \mathbf{w}\right), \\
\boldsymbol{\varphi}_{\mathbf{u}}(T)=0
\end{array}\right.
$$

This theorem is an immediate consequence of Theorem 2.5 and the chain rule. As usual, we have introduced the adjoint state $\boldsymbol{\varphi}_{\mathbf{u}}$ which satisfies the adjoint equation of (2.4). The regularity of $\boldsymbol{\varphi}_{\mathbf{u}}$ follows again from ([29], Thm. 4.2). In [4], this theorem was proved for $\bar{p}=2$.

The existence of a solution of $(\mathrm{P})$ is proved in the standard way. It is enough to use Corollary 2.4. Following again [4], we get the first order necessary conditions.

Theorem 3.3. Let us assume that $\overline{\mathbf{u}}$ is a local solution of problem $(\mathrm{P})$, then there exist $\overline{\mathbf{y}}, \bar{\varphi} \in \mathbf{W}_{\bar{p}}^{2,1}\left(\Omega_{T}\right) \cap$ $C\left([0, T], \mathbf{Y}_{\bar{p}}\right)$ such that

$$
\begin{aligned}
& \left\{\begin{array}{l}
\left(\overline{\mathbf{y}}_{t}, \mathbf{w}\right)+a(\overline{\mathbf{y}}, \mathbf{w})+c(\overline{\mathbf{y}}, \overline{\mathbf{y}}, \mathbf{w})=(\mathbf{f}+\overline{\mathbf{u}}, \mathbf{w}) \quad \forall \mathbf{w} \in \mathbf{Y}_{2}, \\
\overline{\mathbf{y}}(0)=\mathbf{y}_{0},
\end{array}\right. \\
& \left\{\begin{array}{l}
-\left(\overline{\boldsymbol{\varphi}}_{t}, \mathbf{w}\right)+a(\overline{\boldsymbol{\varphi}}, \mathbf{w})+c(\mathbf{w}, \overline{\mathbf{y}}, \overline{\boldsymbol{\varphi}})+c(\overline{\mathbf{y}}, \mathbf{w}, \overline{\boldsymbol{\varphi}})=\left(\overline{\mathbf{y}}-\mathbf{y}_{d}, \mathbf{w}\right) \forall \mathbf{w} \in \mathbf{Y}_{2}, \\
\overline{\boldsymbol{\varphi}}(T)=0
\end{array}\right. \\
& \int_{0}^{T} \int_{\Omega} \bar{\varphi}(\mathbf{u}-\overline{\mathbf{u}}) \mathrm{d} x \mathrm{~d} t \geq 0 \quad \forall \mathbf{u} \in \mathcal{U}_{a d}
\end{aligned}
$$

From (3.6) it follows for almost all $(t, x) \in \Omega_{T}$ and $j=1,2$

$$
\left\{\begin{array} { c l } 
{ \overline { u } _ { j } ( t , x ) = \alpha _ { j } } & { \Rightarrow \overline { \varphi } _ { j } ( t , x ) \geq 0 , } \\
{ \overline { u } _ { j } ( t , x ) = \beta _ { j } } & { \Rightarrow \overline { \varphi } _ { j } ( t , x ) \leq 0 , } \\
{ \alpha _ { j } < \overline { u } _ { j } ( t , x ) < \beta _ { j } } & { \Rightarrow \overline { \varphi } _ { j } ( t , x ) = 0 , }
\end{array} \text { and } \left\{\begin{array}{l}
\bar{\varphi}_{j}(t, x)>0 \Rightarrow \bar{u}_{j}(t, x)=\alpha_{j}, \\
\bar{\varphi}_{j}(t, x)<0 \Rightarrow \bar{u}_{j}(t, x)=\beta_{j},
\end{array}\right.\right.
$$

where $\bar{\varphi}=\left(\bar{\varphi}_{1}, \bar{\varphi}_{2}\right)$. These relations prove that $\overline{\mathbf{u}}$ is a bang-bang control if

$$
\mid\left\{(x, t) \in \Omega_{T}: \bar{\varphi}_{1}(x, t)=0 \text { or } \bar{\varphi}_{2}(x, t)=0\right\} \mid=0,
$$

where $|E|$ denotes the Lebesgue measure of a set $E$. To write the second order conditions we introduce the cone of critical directions

$$
\begin{aligned}
& \mathcal{C}_{\overline{\mathbf{u}}}=\left\{\mathbf{v} \in \mathbf{L}^{2}\left(\Omega_{T}\right): \mathbf{v} \text { satisfies }(3.9)-(3.10) \text { and } J^{\prime}(\overline{\mathbf{u}}) \mathbf{v}=0\right\}, \\
& v_{j}(t, x) \geq 0 \text { if } \bar{u}_{j}(t, x)=\alpha_{j}, \\
& v_{j}(t, x) \leq 0 \text { if } \bar{u}_{j}(t, x)=\beta_{j}, \quad j=1,2 .
\end{aligned}
$$


Remark 3.4. Due to (3.3), we have $J^{\prime}(\overline{\mathbf{u}})=\bar{\varphi}$. Hence, $J^{\prime}(\overline{\mathbf{u}}): \mathbf{L}^{\bar{p}}\left(\Omega_{T}\right) \longrightarrow \mathbb{R}$ can be extended to a continuous linear form $J^{\prime}(\overline{\mathbf{u}}): \mathbf{L}^{2}\left(\Omega_{T}\right) \longrightarrow \mathbb{R}$ in the obvious way. Hence, $\mathcal{C}_{\overline{\mathbf{u}}}$ is well defined. Moreover, from (3.7) we deduce that if $\mathbf{v}$ satisfies (3.9) and (3.10), then $J^{\prime}(\overline{\mathbf{u}}) \mathbf{v}=0$ if and only if $\bar{\varphi}(x, t) \cdot \mathbf{v}(x, t)=0$ for almost every point $(x, t) \in \Omega_{T}$.

Now, we establish the second order necessary conditions.

Theorem 3.5. Let $\overline{\mathbf{u}}$ be a local solution of problem $(\mathrm{P})$, then $J^{\prime \prime}(\overline{\mathbf{u}}) \mathbf{v}^{2} \geq 0 \forall \mathbf{v} \in \mathcal{C}_{\overline{\mathbf{u}}}$.

The proof of this theorem is similar to the one made in ([9], Thm. 3.6) for the case of steady-state NavierStokes equations. However, we cannot proceed as in [9] or [4] to write the sufficient second order conditions. The main difference is that the Tikhonov regularizing term $\|\mathbf{u}\|_{\mathbf{L}^{2}\left(\Omega_{T}\right)}^{2}$ is not included in our cost functional $J$. As a consequence, the condition $J^{\prime \prime}(\overline{\mathbf{u}}) \mathbf{v}^{2}>0$ for all $\mathbf{v} \in \mathcal{C}_{\overline{\mathbf{u}}} \backslash\{0\}$ is not enough to deduce local optimality for $\overline{\mathbf{u}}$. This is usual in infinite dimension optimization problems. In addition, since we have pointwise constraints for the controls, we need to increase the cone of critical directions; see [14]. To this end, for every $\varrho>0$ we consider the extended cone

$$
\mathcal{C} \frac{\varrho}{\overline{\mathbf{u}}}=\left\{\mathbf{v} \in \mathbf{L}^{2}\left(\Omega_{T}\right): \mathbf{v} \text { satisfies }(3.9)--(3.10) \text { and } J^{\prime}(\overline{\mathbf{u}}) \mathbf{v} \leq \varrho\left\|\mathbf{z}_{\mathbf{v}}\right\|_{\mathbf{L}^{2}\left(\Omega_{T}\right)}\right\},
$$

where $\mathbf{z}_{\mathbf{v}}=G^{\prime}(\overline{\mathbf{u}}) \mathbf{v}$ is the solution of (2.4). Observe that, as a consequence of (3.7), for every $\mathbf{v} \in \mathbf{L}^{2}\left(\Omega_{T}\right)$ satisfying (3.9)-(3.10) the inequality $\bar{\varphi}(x, t) \cdot \mathbf{v}(x, t) \geq 0$ holds for almost every point $(x, t) \in \Omega_{T}$. Hence, the condition $J^{\prime}(\overline{\mathbf{u}}) \mathbf{v}=0$ assumed in the definition of $\mathcal{C}_{\overline{\mathbf{u}}}$ is replaced in the definition of $\mathcal{C}_{\overline{\mathbf{u}}}$ by the assumption that $J^{\prime}(\overline{\mathbf{u}}) \mathbf{v}$ is small. Here, $\varrho$ is the parameter controlling how small is $J^{\prime}(\overline{\mathbf{u}}) \mathbf{v}$. Obviously we have that $\mathcal{C}_{\overline{\mathbf{u}}}=\mathcal{C}_{\overline{\mathbf{u}}}^{0}$. Now, the reader can be tempted to assume the second order condition: $J^{\prime \prime}(\overline{\mathbf{u}}) \mathbf{v}^{2} \geq \delta\|\mathbf{v}\|_{\mathbf{L}^{2}\left(\Omega_{T}\right)}^{2}$ for every $\mathbf{v} \in \mathcal{C}_{\overline{\mathbf{u}}}$. However, this condition is not satisfied except maybe for a few extreme cases; see [3]. The reader is referred to $[7,8]$ for additional discussion on the sufficient second order conditions. The following theorem provides the correct second order condition.

Theorem 3.6. Let us assume that $\overline{\mathbf{u}} \in \mathcal{U}_{\text {ad }}$ satisfies (3.4)-(3.6) along with the associated state and adjoint state $(\overline{\mathbf{y}}, \bar{\varphi}) \in\left(\mathbf{W}_{\bar{p}}^{2,1}\left(\Omega_{T}\right) \cap C\left([0, T] ; \mathbf{Y}_{\bar{p}}\right)\right)^{2}$. We also suppose that

$$
\exists \varrho>0 \text { and } \exists \delta>0: J^{\prime \prime}(\overline{\mathbf{u}}) \mathbf{v}^{2} \geq \delta\left\|\mathbf{z}_{\mathbf{v}}\right\|_{\mathbf{L}^{2}\left(\Omega_{T}\right)}^{2} \quad \forall \mathbf{v} \in \mathcal{C} \underline{\underline{\mathbf{u}}} .
$$

Then, there exist $\varepsilon>0$ and $\kappa>0$ such that the following inequality holds

$$
\frac{\kappa}{2}\left\|\mathbf{y}_{\mathbf{u}}-\overline{\mathbf{y}}\right\|_{\mathbf{L}^{2}\left(\Omega_{T}\right)}^{2}+J(\overline{\mathbf{u}}) \leq J(\mathbf{u}), \forall \mathbf{u} \in \mathcal{U}_{a d} \text { with }\|\mathbf{u}-\overline{\mathbf{u}}\|_{\mathbf{L}^{2}\left(\Omega_{T}\right)}<\varepsilon .
$$

The rest of the section is dedicated to the proof of this theorem. In the sequel $(\overline{\mathbf{u}}, \overline{\mathbf{y}}, \bar{\varphi})$ denote the functions introduced in the theorem.

Lemma 3.7. There exist constants $\bar{M}$ and $\tilde{M}$ depending only on $T, \nu$ and $\|\overline{\mathbf{y}}\|_{\mathbf{L} \infty\left(\Omega_{T}\right)}$ such that for every $\mathbf{u} \in \mathcal{U}_{a d}$

$$
\begin{aligned}
\left\|\mathbf{y}_{\mathbf{u}}-\overline{\mathbf{y}}-\mathbf{z}_{\mathbf{u}-\overline{\mathbf{u}}}\right\|_{C\left([0, T] ; \mathbf{L}^{2}(\Omega)\right)} & \leq \bar{M}\left\|\mathbf{y}_{u}-\overline{\mathbf{y}}\right\|_{\mathbf{L}^{\infty}\left(\Omega_{T}\right)}\left\|\mathbf{y}_{\mathbf{u}}-\overline{\mathbf{y}}\right\|_{\mathbf{L}^{2}\left(\Omega_{T}\right)}, \\
\left\|\mathbf{y}_{\mathbf{u}}-\overline{\mathbf{y}}-\mathbf{z}_{\mathbf{u}-\overline{\mathbf{u}}}\right\|_{\mathbf{L}^{2}\left(\Omega_{T}\right)} & \leq \bar{M} \sqrt{T}\left\|\mathbf{y}_{u}-\overline{\mathbf{y}}\right\|_{\mathbf{L}^{\infty}\left(\Omega_{T}\right)}\left\|\mathbf{y}_{\mathbf{u}}-\overline{\mathbf{y}}\right\|_{\mathbf{L}^{2}\left(\Omega_{T}\right)}, \\
\left\|\nabla \mathbf{y}_{\mathbf{u}}-\nabla \overline{\mathbf{y}}-\nabla \mathbf{z}_{\mathbf{u}-\overline{\mathbf{u}}}\right\|_{\mathbf{L}^{2}\left(\Omega_{T}\right)} & \leq \tilde{M}\left\|\mathbf{y}_{u}-\overline{\mathbf{y}}\right\|_{\mathbf{L}^{\infty}\left(\Omega_{T}\right)}\left\|\mathbf{y}_{\mathbf{u}}-\overline{\mathbf{y}}\right\|_{\mathbf{L}^{2}\left(\Omega_{T}\right)} .
\end{aligned}
$$


Proof. Let us define $\mathbf{e}=\mathbf{y}_{\mathbf{u}}-\overline{\mathbf{y}}-\mathbf{z}_{\mathbf{u}-\overline{\mathbf{u}}}$. From (2.1), (3.4) and the equation satisfied by $\mathbf{z}_{\mathbf{u}-\overline{\mathbf{u}}}$

$$
\left\{\begin{array}{l}
\left(\mathbf{z}_{\mathbf{u}-\overline{\mathbf{u}}, t}, \boldsymbol{\psi}\right)+a\left(\mathbf{z}_{\mathbf{u}-\overline{\mathbf{u}}}, \boldsymbol{\psi}\right)+c\left(\mathbf{z}_{\mathbf{u}-\overline{\mathbf{u}}}, \overline{\mathbf{y}}, \boldsymbol{\psi}\right)+c\left(\overline{\mathbf{y}}, \mathbf{z}_{\mathbf{u}-\overline{\mathbf{u}}}, \boldsymbol{\psi}\right)=(\mathbf{u}-\overline{\mathbf{u}}, \boldsymbol{\psi}) \forall \boldsymbol{\psi} \in \mathbf{Y}_{2}, \\
\mathbf{z}(0)=0
\end{array}\right.
$$

and using the identity

$$
c\left(\mathbf{y}_{\mathbf{u}}, \mathbf{y}_{\mathbf{u}}, \boldsymbol{\psi}\right)-c(\overline{\mathbf{y}}, \overline{\mathbf{y}}, \boldsymbol{\psi})-c\left(\mathbf{z}_{\mathbf{u}-\overline{\mathbf{u}}}, \overline{\mathbf{y}}, \boldsymbol{\psi}\right)-c\left(\overline{\mathbf{y}}, \mathbf{z}_{\mathbf{u}-\overline{\mathbf{u}}}, \boldsymbol{\psi}\right)=c(\mathbf{e}, \overline{\mathbf{y}}, \boldsymbol{\psi})+c(\overline{\mathbf{y}}, \mathbf{e}, \boldsymbol{\psi})+c\left(\mathbf{y}_{\mathbf{u}}-\overline{\mathbf{y}}, \mathbf{y}_{\mathbf{u}}-\overline{\mathbf{y}}, \boldsymbol{\psi}\right),
$$

we deduce the equation satisfied by $\mathbf{e}$

$$
\left\{\begin{array}{l}
\left(\mathbf{e}_{t}, \boldsymbol{\psi}\right)+a(\mathbf{e}, \boldsymbol{\psi})+c(\mathbf{e}, \overline{\mathbf{y}}, \boldsymbol{\psi})+c(\overline{\mathbf{y}}, \mathbf{e}, \boldsymbol{\psi})+c\left(\mathbf{y}_{\mathbf{u}}-\overline{\mathbf{y}}, \mathbf{y}_{\mathbf{u}}-\overline{\mathbf{y}}, \boldsymbol{\psi}\right)=0 \forall \boldsymbol{\psi} \in \mathbf{Y}_{2} \\
\mathbf{e}(0)=0
\end{array}\right.
$$

Taking $\boldsymbol{\psi}=\mathbf{e}$ in this equation and integrating in the interval $(0, t)$ we get

$$
\begin{aligned}
& \frac{1}{2}\|\mathbf{e}(t)\|^{2}+\nu \int_{0}^{t}\|\nabla \mathbf{e}\|^{2} \mathrm{~d} s=\frac{1}{2}\|\mathbf{e}(t)\|^{2}+\int_{0}^{t} a(\mathbf{e}, \mathbf{e}) \mathrm{d} s \\
& \leq \int_{0}^{t}|c(\mathbf{e}, \overline{\mathbf{y}}, \mathbf{e})| \mathrm{d} s+\int_{0}^{t}|c(\overline{\mathbf{y}}, \mathbf{e}, \mathbf{e})| \mathrm{d} s+\int_{0}^{t}\left|c\left(\mathbf{y}_{\mathbf{u}}-\overline{\mathbf{y}}, \mathbf{y}_{\mathbf{u}}-\overline{\mathbf{y}}, \mathbf{e}\right)\right| \mathrm{d} s .
\end{aligned}
$$

Using the skew-symmetric property of $c: c(\mathbf{y}, \mathbf{z}, \mathbf{w})=-c(\mathbf{y}, \mathbf{w}, \mathbf{z})$, we obtain that $c(\overline{\mathbf{y}}, \mathbf{e}, \mathbf{e})=0$ and

$$
\int_{0}^{t}|c(\mathbf{e}, \overline{\mathbf{y}}, \mathbf{e})| \mathrm{d} s=\int_{0}^{t}|c(\mathbf{e}, \mathbf{e}, \overline{\mathbf{y}})| \mathrm{d} s \leq\|\overline{\mathbf{y}}\|_{\mathbf{L}^{\infty}\left(\Omega_{T}\right)} \int_{0}^{t}\|\mathbf{e}\|\|\nabla \mathbf{e}\| \mathrm{d} s \leq \frac{1}{\nu}\|\overline{\mathbf{y}}\|_{\mathbf{L}^{\infty}\left(\Omega_{T}\right)}^{2} \int_{0}^{t}\|\mathbf{e}\|^{2} \mathrm{~d} s+\frac{\nu}{4} \int_{0}^{t}\|\nabla \mathbf{e}\|^{2} \mathrm{~d} s .
$$

Now, we estimate the last term of $(3.18)$

$$
\begin{aligned}
\int_{0}^{t}\left|c\left(\mathbf{y}_{\mathbf{u}}-\overline{\mathbf{y}}, \mathbf{y}_{\mathbf{u}}-\overline{\mathbf{y}}, \mathbf{e}\right)\right| \mathrm{d} s & =\int_{0}^{t}\left|c\left(\mathbf{y}_{\mathbf{u}}-\overline{\mathbf{y}}, \mathbf{e}, \mathbf{y}_{\mathbf{u}}-\overline{\mathbf{y}}\right)\right| \mathrm{d} s \\
& \leq\left\|\mathbf{y}_{\mathbf{u}}-\overline{\mathbf{y}}\right\|_{\mathbf{L} \infty\left(\Omega_{T}\right)} \int_{0}^{t}\left\|\mathbf{y}_{\mathbf{u}}-\overline{\mathbf{y}}\right\|\|\nabla \mathbf{e}\| \mathrm{d} s \\
& \leq \frac{1}{\nu}\left\|\mathbf{y}_{\mathbf{u}}-\overline{\mathbf{y}}\right\|_{\mathbf{L}^{\infty}\left(\Omega_{T}\right)}^{2}\left\|\mathbf{y}_{\mathbf{u}}-\overline{\mathbf{y}}\right\|_{\mathbf{L}^{2}\left(\Omega_{T}\right)}^{2}+\frac{\nu}{4} \int_{0}^{t}\|\nabla \mathbf{e}\|^{2} \mathrm{~d} s .
\end{aligned}
$$

From (3.18)-(3.20) we infer with Gronwall's inequality for every $t \in[0, T]$

$$
\|\mathbf{e}(t)\|^{2} \leq \frac{2}{\nu} \exp \left(\frac{2 T}{\nu}\|\overline{\mathbf{y}}\|_{\mathbf{L}^{\infty}\left(\Omega_{T}\right)}^{2}\right)\left\|\mathbf{y}_{\mathbf{u}}-\overline{\mathbf{y}}\right\|_{\mathbf{L}^{\infty}\left(\Omega_{T}\right)}^{2}\left\|\mathbf{y}_{\mathbf{u}}-\overline{\mathbf{y}}\right\|_{\mathbf{L}^{2}\left(\Omega_{T}\right)}^{2} .
$$

This implies (3.14) with

$$
\bar{M}=\sqrt{\frac{2}{\nu}} \exp \left(\frac{T}{\nu}\|\overline{\mathbf{y}}\|_{\mathbf{L}^{\infty}\left(\Omega_{T}\right)}\right) .
$$

Inequality (3.15) is a straightforward consequence of (3.14). Now, (3.16) is deduced from (3.18)-(3.20) and (3.15) taking $t=T$ and setting

$$
\tilde{M}=\frac{\sqrt{2}}{\nu} \sqrt{\|\overline{\mathbf{y}}\|_{\mathbf{L}^{\infty}\left(\Omega_{T}\right)}^{2} T \bar{M}^{2}+1}
$$


Lemma 3.8. Let $\bar{M}$ be the constant introduced in Lemma 3.7. Let $\varepsilon>0$ satisfy $0<\varepsilon \leq \frac{1-\frac{1}{\sqrt{2}}}{\bar{M} \sqrt{T}}$. Then, the following inequality holds for every $\mathbf{u} \in \mathcal{U}_{\text {ad }}$ such that $\left\|\mathbf{y}_{\mathbf{u}}-\overline{\mathbf{y}}\right\|_{\mathbf{L}^{\infty}\left(\Omega_{T}\right)} \leq \varepsilon$

$$
\left\|\mathbf{y}_{\mathbf{u}}-\overline{\mathbf{y}}\right\|_{\mathbf{L}^{2}\left(\Omega_{T}\right)} \leq \sqrt{2}\left\|\mathbf{z}_{\mathbf{u}-\overline{\mathbf{u}}}\right\|_{\mathbf{L}^{2}\left(\Omega_{T}\right)} .
$$

Proof. From (3.15) we get

$$
\begin{aligned}
\left\|\mathbf{y}_{\mathbf{u}}-\overline{\mathbf{y}}\right\|_{\mathbf{L}^{2}\left(\Omega_{T}\right)} & \leq\left\|\mathbf{y}_{\mathbf{u}}-\overline{\mathbf{y}}-\mathbf{z}_{\mathbf{u}-\overline{\mathbf{u}}}\right\|_{\mathbf{L}^{2}\left(\Omega_{T}\right)}+\left\|\mathbf{z}_{\mathbf{u}-\overline{\mathbf{u}}}\right\|_{\mathbf{L}^{2}\left(\Omega_{T}\right)} \\
& \leq \bar{M} \sqrt{T} \varepsilon\left\|\mathbf{y}_{\mathbf{u}}-\overline{\mathbf{y}}\right\|_{\mathbf{L}^{2}\left(\Omega_{T}\right)}+\left\|\mathbf{z}_{\mathbf{u}-\overline{\mathbf{u}}}\right\|_{\mathbf{L}^{2}\left(\Omega_{T}\right)} .
\end{aligned}
$$

From this inequality and the assumption on $\varepsilon$ we get

$$
\left\|\mathbf{y}_{\mathbf{u}}-\overline{\mathbf{y}}\right\|_{\mathbf{L}^{2}\left(\Omega_{T}\right)} \leq \frac{1}{1-\bar{M} \sqrt{T} \varepsilon}\left\|\mathbf{z}_{\mathbf{u}-\overline{\mathbf{u}}}\right\|_{\mathbf{L}^{2}\left(\Omega_{T}\right)} \leq \sqrt{2}\left\|\mathbf{z}_{\mathbf{u}-\overline{\mathbf{u}}}\right\|_{\mathbf{L}^{2}\left(\Omega_{T}\right)} .
$$

Lemma 3.9. There exist constants $K_{\boldsymbol{\alpha}, \boldsymbol{\beta}}$ and $\tilde{K}_{\boldsymbol{\alpha}, \boldsymbol{\beta}}$ such that for all $\mathbf{v} \in \mathbf{L}^{2}\left(\Omega_{T}\right)$ and every $\mathbf{u} \in \mathcal{U}_{a d}$ the following inequalities hold

$$
\begin{aligned}
\left\|\mathbf{z}_{\mathbf{u}, \mathbf{v}}-\mathbf{z}_{\mathbf{v}}\right\|_{C\left([0, T] ; \mathbf{L}^{2}(\Omega)\right)} & \leq K_{\boldsymbol{\alpha}, \boldsymbol{\beta}}\left\|\mathbf{y}_{\mathbf{u}}-\overline{\mathbf{y}}\right\|_{\mathbf{L}^{\infty}\left(\Omega_{T}\right)}\left\|\mathbf{z}_{\mathbf{v}}\right\|_{\mathbf{L}^{2}\left(\Omega_{T}\right)}, \\
\left\|\nabla \mathbf{z}_{\mathbf{u}, \mathbf{v}}-\nabla \mathbf{z}_{\mathbf{v}}\right\|_{\mathbf{L}^{2}\left(\Omega_{T}\right)} & \leq \tilde{K} \boldsymbol{\alpha}, \boldsymbol{\beta}\left\|\mathbf{y}_{\mathbf{u}}-\overline{\mathbf{y}}\right\|_{\mathbf{L}^{\infty}\left(\Omega_{T}\right)}\left\|\mathbf{z}_{\mathbf{v}}\right\|_{\mathbf{L}^{2}\left(\Omega_{T}\right)}, \\
\left\|\mathbf{z}_{\mathbf{u}, \mathbf{v}}\right\|_{\mathbf{L}^{2}\left(\Omega_{T}\right)} & \leq\left(1+\sqrt{T} K_{\boldsymbol{\alpha}, \boldsymbol{\beta}}\left\|\mathbf{y}_{\mathbf{u}}-\overline{\mathbf{y}}\right\|_{\mathbf{L}^{\infty}\left(\Omega_{T}\right)}\right)\left\|\mathbf{z}_{\mathbf{V}}\right\|_{\mathbf{L}^{2}\left(\Omega_{T}\right)},
\end{aligned}
$$

where $\mathbf{z}_{\mathbf{v}}=G^{\prime}(\overline{\mathbf{u}}) \mathbf{v}$ and $\mathbf{z}_{\mathbf{u}, \mathbf{v}}=G^{\prime}(\mathbf{u}) \mathbf{v}$.

Proof. According to Theorem 2.5, $\mathbf{z}_{\mathbf{v}}$ and $\mathbf{z}_{\mathbf{u}, \mathbf{v}}$ satisfy the variational equations

$$
\left\{\begin{array}{l}
\left(\mathbf{z}_{\mathbf{v}, t}, \boldsymbol{\psi}\right)+a\left(\mathbf{z}_{\mathbf{v}}, \boldsymbol{\psi}\right)+c\left(\mathbf{z}_{\mathbf{v}}, \overline{\mathbf{y}}, \boldsymbol{\psi}\right)+c\left(\overline{\mathbf{y}}, \mathbf{z}_{\mathbf{v}}, \boldsymbol{\psi}\right)=(\mathbf{v}, \boldsymbol{\psi}) \forall \boldsymbol{\psi} \in \mathbf{Y}_{2}, \\
\mathbf{z}_{\mathbf{v}}(0)=0
\end{array}\right.
$$

and

$$
\left\{\begin{array}{l}
\left(\mathbf{z}_{\mathbf{u}, \mathbf{v}, t}, \boldsymbol{\psi}\right)+a\left(\mathbf{z}_{\mathbf{u}, \mathbf{v}}, \boldsymbol{\psi}\right)+c\left(\mathbf{z}_{\mathbf{u}, \mathbf{v}}, \mathbf{y}_{\mathbf{u}}, \boldsymbol{\psi}\right)+c\left(\mathbf{y}_{\mathbf{u}}, \mathbf{z}_{\mathbf{u}, \mathbf{v}}, \boldsymbol{\psi}\right)=(\mathbf{v}, \boldsymbol{\psi}) \forall \boldsymbol{\psi} \in \mathbf{Y}_{2}, \\
\mathbf{z}_{\mathbf{u}, \mathbf{v}}(0)=0 .
\end{array}\right.
$$

Setting $\mathbf{e}=\mathbf{z}_{\mathbf{u}, \mathbf{v}}-\mathbf{z}_{\mathbf{v}}, \boldsymbol{\psi}=\mathbf{e}$, subtracting the above equations and using that

$$
\begin{aligned}
c\left(\mathbf{y}_{\mathbf{u}}, \mathbf{z}_{\mathbf{u}, \mathbf{v}}, \boldsymbol{\psi}\right)+c\left(\mathbf{z}_{\mathbf{u}, \mathbf{v}}, \mathbf{y}_{\mathbf{u}}, \boldsymbol{\psi}\right)-c\left(\overline{\mathbf{y}}, \mathbf{z}_{\mathbf{v}}, \boldsymbol{\psi}\right)-c\left(\mathbf{z}_{\mathbf{v}}, \overline{\mathbf{y}}, \boldsymbol{\psi}\right)= & c\left(\mathbf{y}_{\mathbf{u}}, \mathbf{e}, \boldsymbol{\psi}\right)+c\left(\mathbf{e}, \mathbf{y}_{\mathbf{u}}, \boldsymbol{\psi}\right) \\
& +c\left(\mathbf{y}_{\mathbf{u}}-\overline{\mathbf{y}}, \mathbf{z}_{\mathbf{v}}, \boldsymbol{\psi}\right)+c\left(\mathbf{z}_{\mathbf{v}}, \mathbf{y}_{\mathbf{u}}-\overline{\mathbf{y}}, \boldsymbol{\psi}\right),
\end{aligned}
$$

and $c\left(\mathbf{y}_{\mathbf{u}}, \mathbf{e}, \mathbf{e}\right)=0$, we get

$$
\left\{\begin{array}{l}
\left(\mathbf{e}_{t}, \mathbf{e}\right)+a(\mathbf{e}, \mathbf{e})+c\left(\mathbf{e}, \mathbf{y}_{\mathbf{u}}, \mathbf{e}\right)+c\left(\mathbf{y}_{\mathbf{u}}-\overline{\mathbf{y}}, \mathbf{z}_{\mathbf{v}}, \mathbf{e}\right)+c\left(\mathbf{z}_{\mathbf{v}}, \mathbf{y}_{\mathbf{u}}-\overline{\mathbf{y}}, \mathbf{e}\right)=0 \\
\mathbf{z}_{\mathbf{v}}(0)=0
\end{array}\right.
$$

Integrating the above equation in $(0, t)$ we obtain

$$
\begin{aligned}
\frac{1}{2}\|\mathbf{e}(t)\|^{2}+\nu \int_{0}^{t}\|\nabla \mathbf{e}\|^{2} \mathrm{~d} s & =\frac{1}{2}\|\mathbf{e}(t)\|^{2}+\int_{0}^{t} a(\mathbf{e}, \mathbf{e}) \mathrm{d} s \\
& \leq \int_{0}^{t}\left|c\left(\mathbf{e}, \mathbf{y}_{\mathbf{u}}, \mathbf{e}\right)\right| \mathrm{d} s+\int_{0}^{t}\left|c\left(\mathbf{y}_{\mathbf{u}}-\overline{\mathbf{y}}, \mathbf{z}_{\mathbf{v}}, \mathbf{e}\right)\right| \mathrm{d} s+\int_{0}^{t}\left|c\left(\mathbf{z}_{\mathbf{v}}, \mathbf{y}_{\mathbf{u}}-\overline{\mathbf{y}}, \mathbf{e}\right)\right| \mathrm{d} s .
\end{aligned}
$$


From (2.3) we deduce that

$$
\left\|\mathbf{y}_{\mathbf{u}}\right\|_{\mathbf{L}^{\infty}\left(\Omega_{T}\right)} \leq C_{\boldsymbol{\alpha}, \boldsymbol{\beta}}=\sup \left\{M_{\boldsymbol{\alpha}, \boldsymbol{\beta}}\left(\|\mathbf{f}+\mathbf{u}\|_{\mathbf{L}^{\bar{p}}\left(\Omega_{T}\right)}+\left\|\mathbf{y}_{0}\right\|_{\mathbf{W}^{2-\frac{2}{\bar{p}}, \bar{p}}(\Omega)}\right): \mathbf{u} \in \mathcal{U}_{a d}\right\} .
$$

Using this estimate and the skew-symmetric property of $c$ the following inequalities hold

$$
\int_{0}^{t}\left|c\left(\mathbf{e}, \mathbf{y}_{\mathbf{u}}, \mathbf{e}\right)\right| \mathrm{d} s \leq C_{\boldsymbol{\alpha}, \boldsymbol{\beta}} \int_{0}^{t}\|\mathbf{e}\|\|\nabla \mathbf{e}\| \mathrm{d} s \leq \frac{3}{2 \nu} C_{\boldsymbol{\alpha}, \boldsymbol{\beta}}^{2} \int_{0}^{t}\|\mathbf{e}\|^{2} \mathrm{~d} s+\frac{\nu}{6} \int_{0}^{t}\|\nabla \mathbf{e}\|^{2} \mathrm{~d} s .
$$

Now, we proceed with the second estimate

$$
\begin{aligned}
\int_{0}^{t}\left|c\left(\mathbf{y}_{\mathbf{u}}-\overline{\mathbf{y}}, \mathbf{z}_{\mathbf{v}}, \mathbf{e}\right)\right| \mathrm{d} s & \leq\left\|\mathbf{y}_{\mathbf{u}}-\overline{\mathbf{y}}\right\|_{\mathbf{L}^{\infty}\left(\Omega_{T}\right)} \int_{0}^{t}\left\|\mathbf{z}_{\mathbf{v}}\right\|\|\nabla \mathbf{e}\| \mathrm{d} s \\
& \leq \frac{3}{2 \nu}\left\|\mathbf{y}_{\mathbf{u}}-\overline{\mathbf{y}}\right\|_{\mathbf{L}^{\infty}\left(\Omega_{T}\right)}^{2}\left\|\mathbf{z}_{\mathbf{v}}\right\|_{\mathbf{L}^{2}\left(\Omega_{T}\right)}^{2}+\frac{\nu}{6} \int_{0}^{t}\|\nabla \mathbf{e}\|^{2} \mathrm{~d} s .
\end{aligned}
$$

The same estimate holds for the third right hand term of (3.25). Inserting these estimates in (3.25) we infer

$$
\|\mathbf{e}(t)\|^{2}+\nu \int_{0}^{t}\|\nabla \mathbf{e}\|^{2} \mathrm{~d} s \leq \frac{6}{\nu}\left\|\mathbf{y}_{\mathbf{u}}-\overline{\mathbf{y}}\right\|_{\mathbf{L}^{\infty}\left(\Omega_{T}\right)}^{2}\left\|\mathbf{z}_{\mathbf{v}}\right\|_{\mathbf{L}^{2}\left(\Omega_{T}\right)}^{2}+\frac{3}{\nu} C_{\boldsymbol{\alpha}, \boldsymbol{\beta}}^{2} \int_{0}^{t}\|\mathbf{e}\|^{2} \mathrm{~d} s .
$$

Now, Gronwall's inequality implies

$$
\|\mathbf{e}(t)\|^{2} \leq K_{\boldsymbol{\alpha}, \boldsymbol{\beta}}^{2}\left\|\mathbf{y}_{\mathbf{u}}-\overline{\mathbf{y}}\right\|_{\mathbf{L}^{\infty}\left(\Omega_{T}\right)}^{2}\left\|\mathbf{z}_{\mathbf{v}}\right\|_{\mathbf{L}^{2}\left(\Omega_{T}\right)}^{2},
$$

where

$$
K_{\boldsymbol{\alpha}, \boldsymbol{\beta}}=\sqrt{\frac{6}{\nu}} \exp \left(\frac{3 T C_{\boldsymbol{\alpha}, \boldsymbol{\beta}}^{2}}{2 \nu}\right) .
$$

This inequality leads immediately to (3.22). Moreover, if we insert this inequality in (3.27) and take $t=T$, we deduce

$$
\int_{0}^{T}\|\nabla \mathbf{e}\|^{2} \mathrm{~d} t \leq \frac{3}{\nu^{2}}\left(2+C_{\boldsymbol{\alpha}, \boldsymbol{\beta}^{2}}^{2} K_{\boldsymbol{\alpha}, \boldsymbol{\beta}^{T}}^{T}\right)\left\|\mathbf{y}_{\mathbf{u}}-\overline{\mathbf{y}}\right\|_{\mathbf{L}^{\infty}\left(\Omega_{T}\right)}^{2}\left\|\mathbf{z}_{\mathbf{V}}\right\|_{\mathbf{L}^{2}\left(\Omega_{T}\right)}^{2} .
$$

This implies (3.23) with

$$
\tilde{K}_{\boldsymbol{\alpha}, \boldsymbol{\beta}}=\frac{\sqrt{3}}{\nu} \sqrt{2+C_{\boldsymbol{\alpha}, \boldsymbol{\beta}^{K}}^{2} \boldsymbol{\alpha}, \boldsymbol{\beta}^{T}}
$$

The estimate (3.24) follows from (3.22) and the triangle inequality

$$
\left\|\mathbf{z}_{\mathbf{u}, \mathbf{v}}\right\|_{\mathbf{L}^{2}\left(\Omega_{T}\right)} \leq\left\|\mathbf{z}_{\mathbf{u}, \mathbf{v}}-\mathbf{z}_{\mathbf{v}}\right\|_{\mathbf{L}^{2}\left(\Omega_{T}\right)}+\left\|\mathbf{z}_{\mathbf{v}}\right\|_{\mathbf{L}^{2}\left(\Omega_{T}\right)} .
$$

Lemma 3.10. There exist a constant $D_{\boldsymbol{\alpha}, \boldsymbol{\beta}}$ such that the following estimates hold $\forall \mathbf{u} \in \mathcal{U}_{a d}$

$$
\begin{aligned}
\left\|\nabla \mathbf{z}_{\mathbf{u}-\overline{\mathbf{u}}}\right\|_{\mathbf{L}^{2}\left(\Omega_{T}\right)} & \leq D_{\boldsymbol{\alpha}, \boldsymbol{\beta}}\|\mathbf{u}-\overline{\mathbf{u}}\|_{\mathbf{L}^{2}\left(\Omega_{T}\right)}^{1 / 2}\left\|\mathbf{y}_{\mathbf{u}}-\overline{\mathbf{y}}\right\|_{\mathbf{L}^{2}\left(\Omega_{T}\right)}^{1 / 2}, \\
\left\|\nabla \mathbf{y}_{\mathbf{u}}-\nabla \overline{\mathbf{y}}\right\|_{\mathbf{L}^{2}\left(\Omega_{T}\right)} & \leq\left(\sqrt{L_{2}} L_{\infty} \tilde{M}\|\mathbf{u}-\overline{\mathbf{u}}\|_{\mathbf{L}^{2}\left(\Omega_{T}\right)}^{\frac{2}{\bar{p}}}+D_{\boldsymbol{\alpha}, \boldsymbol{\beta}}\right)\|\mathbf{u}-\overline{\mathbf{u}}\|_{\mathbf{L}^{2}\left(\Omega_{T}\right)}^{1 / 2}\left\|\mathbf{y}_{\mathbf{u}}-\overline{\mathbf{y}}\right\|_{\mathbf{L}^{2}\left(\Omega_{T}\right)}^{1 / 2},
\end{aligned}
$$

where $\tilde{M}, L_{2}$ and $L_{\infty}$ are as in Lemma 3.7 and Corollary 2.6, respectively. 
Proof. In the variational equation satisfied by $\mathbf{z}_{\mathbf{u}-\overline{\mathbf{u}}}$ we take $\boldsymbol{\psi}=\mathbf{z}_{\mathbf{u}-\overline{\mathbf{u}}}$, we make the integral in $(0, t)$ and we use that $c\left(\overline{\mathbf{y}}, \mathbf{z}_{\mathbf{u}-\overline{\mathbf{u}}}, \mathbf{z}_{\mathbf{u}-\overline{\mathbf{u}}}\right)=0$ to deduce

$$
\begin{aligned}
& \frac{1}{2}\left\|\mathbf{z}_{\mathbf{u}-\overline{\mathbf{u}}}(t)\right\|^{2}+\nu \int_{0}^{t}\left\|\nabla \mathbf{z}_{\mathbf{u}-\overline{\mathbf{u}}}\right\|^{2} \mathrm{~d} s \leq \int_{0}^{t}\left|\left(\mathbf{u}-\overline{\mathbf{u}}, \mathbf{z}_{\mathbf{u}-\overline{\mathbf{u}}}\right)\right| \mathrm{d} s+\int_{0}^{t}\left|c\left(\mathbf{z}_{\mathbf{u}-\overline{\mathbf{u}}}, \overline{\mathbf{y}}, \mathbf{z}_{\mathbf{u}-\overline{\mathbf{u}}}\right)\right| \mathrm{d} s \\
& \leq \int_{0}^{t}\left|\left(\mathbf{u}-\overline{\mathbf{u}}, \mathbf{y}_{\mathbf{u}}-\overline{\mathbf{y}}-\mathbf{z}_{\mathbf{u}-\overline{\mathbf{u}}}\right)\right| \mathrm{d} s+\int_{0}^{t}\left|\left(\mathbf{u}-\overline{\mathbf{u}}, \mathbf{y}_{\mathbf{u}}-\overline{\mathbf{y}}\right)\right| \mathrm{d} s+\int_{0}^{t}\left|c\left(\mathbf{z}_{\mathbf{u}-\overline{\mathbf{u}}}, \mathbf{z}_{\mathbf{u}-\overline{\mathbf{u}}}, \overline{\mathbf{y}}\right)\right| \mathrm{d} s .
\end{aligned}
$$

Inserting the estimate (3.14) in the above inequality, we obtain

$$
\begin{aligned}
\frac{1}{2}\left\|\mathbf{z}_{\mathbf{u}-\overline{\mathbf{u}}}(t)\right\|^{2}+\nu \int_{0}^{t}\left\|\nabla \mathbf{z}_{\mathbf{u}-\overline{\mathbf{u}}}\right\|^{2} \mathrm{~d} s \leq & \|\mathbf{u}-\overline{\mathbf{u}}\|_{\mathbf{L}^{2}\left(\Omega_{T}\right)} \bar{M}\left\|\mathbf{y}_{\mathbf{u}}-\overline{\mathbf{y}}\right\|_{\mathbf{L}^{\infty}\left(\Omega_{T}\right)}\left\|\mathbf{y}_{\mathbf{u}}-\overline{\mathbf{y}}\right\|_{\mathbf{L}^{2}\left(\Omega_{T}\right)} \\
& +\|\mathbf{u}-\overline{\mathbf{u}}\|_{\mathbf{L}^{2}\left(\Omega_{T}\right)}\left\|\mathbf{y}_{\mathbf{u}}-\overline{\mathbf{y}}\right\|_{\mathbf{L}^{2}\left(\Omega_{T}\right)}+\|\overline{\mathbf{y}}\|_{\mathbf{L}^{\infty}\left(\Omega_{T}\right)} \int_{0}^{t}\left\|\mathbf{z}_{\mathbf{u}-\overline{\mathbf{u}}}\right\|\left\|\nabla \mathbf{z}_{\mathbf{u}-\overline{\mathbf{u}}}\right\| \mathrm{d} s \\
\leq & \left(1+\bar{M}\left\|\mathbf{y}_{\mathbf{u}}-\overline{\mathbf{y}}\right\|_{\mathbf{L}^{\infty}\left(\Omega_{T}\right)}\right)\|\mathbf{u}-\overline{\mathbf{u}}\|_{\mathbf{L}^{2}\left(\Omega_{T}\right)}\left\|\mathbf{y}_{\mathbf{u}}-\overline{\mathbf{y}}\right\|_{\mathbf{L}^{2}\left(\Omega_{T}\right)} \\
& +\frac{1}{\nu}\|\overline{\mathbf{y}}\|_{\mathbf{L}^{\infty}\left(\Omega_{T}\right)}^{2} \int_{0}^{t}\left\|\mathbf{z}_{\mathbf{u}-\overline{\mathbf{u}}}\right\|^{2} \mathrm{~d} s+\frac{\nu}{2} \int_{0}^{t}\left\|\nabla \mathbf{z}_{\mathbf{u}-\overline{\mathbf{u}}}\right\|^{2} \mathrm{~d} s .
\end{aligned}
$$

From this inequality we infer

$$
\left\|\mathbf{z}_{\mathbf{u}-\overline{\mathbf{u}}}(t)\right\|^{2}+\nu \int_{0}^{t}\left\|\nabla \mathbf{z}_{\mathbf{u}-\overline{\mathbf{u}}}\right\|^{2} \mathrm{~d} s \leq C_{\boldsymbol{\alpha}, \boldsymbol{\beta}}\|\mathbf{u}-\overline{\mathbf{u}}\|_{\mathbf{L}^{2}\left(\Omega_{T}\right)}\left\|\mathbf{y}_{\mathbf{u}}-\overline{\mathbf{y}}\right\|_{\mathbf{L}^{2}\left(\Omega_{T}\right)}+\frac{2}{\nu}\|\overline{\mathbf{y}}\|_{\mathbf{L}^{\infty}\left(\Omega_{T}\right)}^{2} \int_{0}^{t}\left\|\mathbf{z}_{\mathbf{u}-\overline{\mathbf{u}}}\right\|^{2} \mathrm{~d} s,
$$

where

$$
C_{\boldsymbol{\alpha}, \boldsymbol{\beta}}=\max \left\{2\left(1+\bar{M} L_{\infty}\|\mathbf{u}-\overline{\mathbf{u}}\|_{\mathbf{L}^{2}\left(\Omega_{T}\right)}^{\frac{2}{p}}\right): \mathbf{u} \in \mathcal{U}_{a d}\right\} ;
$$

see (2.6). Now, from (3.14) we deduce

$$
\left\|\mathbf{z}_{\mathbf{u}-\overline{\mathbf{u}}}(t)\right\|_{\mathbf{L}^{2}(\Omega)} \leq \bar{M}\left\|\mathbf{y}_{\mathbf{u}}-\overline{\mathbf{y}}\right\|_{\mathbf{L}^{\infty}\left(\Omega_{T}\right)}\left\|\mathbf{y}_{\mathbf{u}}-\overline{\mathbf{y}}\right\|_{\mathbf{L}^{2}\left(\Omega_{T}\right)}+\left\|\mathbf{y}_{\mathbf{u}}(t)-\overline{\mathbf{y}}(t)\right\|_{\mathbf{L}^{2}(\Omega)} \quad \forall t \in[0, T] .
$$

Inserting this estimate in (3.30) and taking $t=T$ we conclude

$$
\begin{aligned}
\nu\left\|\nabla \mathbf{z}_{\mathbf{u}-\overline{\mathbf{u}}}\right\|_{\mathbf{L}^{2}\left(\Omega_{T}\right)}^{2} \leq & C_{\boldsymbol{\alpha}, \boldsymbol{\beta}}\|\mathbf{u}-\overline{\mathbf{u}}\|_{\mathbf{L}^{2}\left(\Omega_{T}\right)}\left\|\mathbf{y}_{\mathbf{u}}-\overline{\mathbf{y}}\right\|_{\mathbf{L}^{2}\left(\Omega_{T}\right)} \\
& +\frac{4}{\nu}\|\overline{\mathbf{y}}\|_{\mathbf{L}^{\infty}\left(\Omega_{T}\right)}^{2}\left[\bar{M}^{2} T\left\|\mathbf{y}_{\mathbf{u}}-\overline{\mathbf{y}}\right\|_{\mathbf{L}^{\infty}\left(\Omega_{T}\right)}^{2}+1\right]\left\|\mathbf{y}_{\mathbf{u}}-\overline{\mathbf{y}}\right\|_{\mathbf{L}^{2}\left(\Omega_{T}\right)}^{2},
\end{aligned}
$$

which with (2.6)and (2.7) proves (3.28). The estimate (3.29) is an immediate consequence of (2.7), (3.16) and (3.28).

Lemma 3.11. There exists a constant $E_{\boldsymbol{\alpha}, \boldsymbol{\beta}}$ such that the following estimate holds

$$
\left\|\boldsymbol{\varphi}_{\mathbf{u}}-\bar{\varphi}\right\|_{L^{\infty}\left(0, T ; \mathbf{H}_{0}^{1}(\Omega)\right)} \leq E_{\boldsymbol{\alpha}, \boldsymbol{\beta}}\left\|\mathbf{y}_{\mathbf{u}}-\overline{\mathbf{y}}\right\|_{\mathbf{L}^{2}\left(\Omega_{T}\right)}^{1 / 2} \quad \forall \mathbf{u} \in \mathcal{U}_{a d}
$$

where $\boldsymbol{\varphi}_{\mathbf{u}}$ is the solution of (3.3).

Proof. First we recall that (3.3) is the variational formulation of the problem

$$
\left\{\begin{array}{l}
-\boldsymbol{\varphi}_{\mathbf{u} t}-\nu \Delta \boldsymbol{\varphi}_{\mathbf{u}}-\left(\mathbf{y}_{\mathbf{u}} \cdot \nabla\right) \boldsymbol{\varphi}_{\mathbf{u}}+\left(\nabla \mathbf{y}_{\mathbf{u}}\right)^{T} \boldsymbol{\varphi}_{\mathbf{u}}+\nabla \pi_{\mathbf{u}}=\mathbf{y}_{\mathbf{u}}-\mathbf{y}_{d} \text { in } \Omega_{T} \\
\operatorname{div} \boldsymbol{\varphi}_{\mathbf{u}}=0 \text { in } \Omega_{T}, \boldsymbol{\varphi}_{\mathbf{u}}(T)=0 \text { in } \Omega, \varphi_{\mathbf{u}}=0 \text { on } \Sigma_{T}
\end{array}\right.
$$


Usin again ([29], Thm. 4.2) and taking into account (2.2) and that $\mathbf{y}_{d} \in \mathbf{L}^{4}\left(\Omega_{T}\right)$, we deduce the existence of a constant $C_{\boldsymbol{\alpha}, \boldsymbol{\beta}}$ such that

$$
\left\|\boldsymbol{\varphi}_{\mathbf{u}}\right\|_{\mathbf{W}_{4}^{2,1}\left(\Omega_{T}\right)} \leq C_{\boldsymbol{\alpha}, \boldsymbol{\beta}} \quad \forall \mathbf{u} \in \mathcal{U}_{a d}
$$

We have the analogous equation for $\bar{\varphi}$

$$
\left\{\begin{array}{l}
-\overline{\boldsymbol{\varphi}}_{t}-\nu \Delta \overline{\boldsymbol{\varphi}}-(\overline{\mathbf{y}} \cdot \nabla) \overline{\boldsymbol{\varphi}}+(\nabla \overline{\mathbf{y}})^{T} \overline{\boldsymbol{\varphi}}+\nabla \bar{\pi}=\overline{\mathbf{y}}-\mathbf{y}_{d} \text { in } \Omega_{T} \\
\operatorname{div} \bar{\varphi}=0 \text { in } \Omega_{T}, \bar{\varphi}(T)=0 \text { in } \Omega, \bar{\varphi}=0 \text { on } \Sigma_{T}
\end{array}\right.
$$

Setting $\mathbf{e}=\varphi_{\mathbf{u}}-\bar{\varphi}$ and subtracting (3.34) to (3.32) it follows

$$
\left\{\begin{array}{l}
-\mathbf{e}_{t}-\nu \Delta \mathbf{e}-(\overline{\mathbf{y}} \cdot \nabla) \mathbf{e}+(\nabla \overline{\mathbf{y}})^{T} \mathbf{e}+\nabla \pi \\
=\mathbf{y}_{\mathbf{u}}-\overline{\mathbf{y}}+\left[\left(\mathbf{y}_{\mathbf{u}}-\overline{\mathbf{y}}\right) \cdot \nabla\right] \boldsymbol{\varphi}_{\mathbf{u}}-\left(\nabla \mathbf{y}_{\mathbf{u}}-\nabla \overline{\mathbf{y}}\right)^{T} \boldsymbol{\varphi}_{\mathbf{u}} \text { in } \Omega_{T} \\
\operatorname{div} \mathbf{e}=0 \text { in } \Omega_{T}, \mathbf{e}(T)=0 \text { in } \Omega, \mathbf{e}=0 \text { on } \Sigma_{T}
\end{array}\right.
$$

Now, we have that $\mathbf{e} \in \mathbf{H}^{2,1}\left(\Omega_{T}\right) \cap C\left([0, T], \mathbf{Y}_{2}\right)$ and the norm of $\mathbf{e}$ in $L^{\infty}\left(0, T ; \mathbf{H}_{0}^{1}(\Omega)\right)$ is estimated by the $\mathbf{L}^{2}\left(\Omega_{T}\right)$ norm of the right hand side of (3.35) multiplied by a constant only depending on $\overline{\mathbf{y}}, \nu$ and $T$. To prove (3.31) we have to estimate the three terms appearing to the right of the equation (3.35). The first estimate is obvious with $(2.7)$

$$
\left\|\mathbf{y}_{\mathbf{u}}-\overline{\mathbf{y}}\right\|_{\mathbf{L}^{2}\left(\Omega_{T}\right)} \leq \sqrt{L_{2}}\|\mathbf{u}-\overline{\mathbf{u}}\|_{\mathbf{L}^{2}\left(\Omega_{T}\right)}^{\frac{1}{2}}\left\|\mathbf{y}_{\mathbf{u}}-\overline{\mathbf{y}}\right\|_{\mathbf{L}^{2}\left(\Omega_{T}\right)}^{1 / 2}
$$

For the second term we proceed as follows

$$
\begin{aligned}
& \left\|\left[\left(\mathbf{y}_{\mathbf{u}}-\overline{\mathbf{y}}\right) \cdot \nabla\right] \boldsymbol{\varphi}_{\mathbf{u}}\right\|_{\mathbf{L}^{2}\left(\Omega_{T}\right)} \leq\left(\int_{0}^{T}\left\|\nabla \boldsymbol{\varphi}_{\mathbf{u}}\right\|_{\mathbf{L}^{\infty}(\Omega)}^{2}\left\|\mathbf{y}_{\mathbf{u}}-\overline{\mathbf{y}}\right\|_{\mathbf{L}^{2}(\Omega)}^{2} \mathrm{~d} t\right)^{1 / 2} \\
& \leq C_{\Omega}\left(\int_{0}^{T}\left\|\boldsymbol{\varphi}_{\mathbf{u}}\right\|_{\mathbf{W}^{2,4}(\Omega)}^{2}\left\|\mathbf{y}_{\mathbf{u}}-\overline{\mathbf{y}}\right\|_{\mathbf{L}^{2}(\Omega)}^{2} \mathrm{~d} t\right)^{1 / 2} \\
& \leq C_{\Omega}\left\|\boldsymbol{\varphi}_{\mathbf{u}}\right\|_{L^{4}\left(0, T ; \mathbf{W}^{2,4}(\Omega)\right)}\left\|\mathbf{y}_{\mathbf{u}}-\overline{\mathbf{y}}\right\|_{\mathbf{L}^{4}\left(\Omega_{T}\right)} \\
& \leq \sqrt{2} C_{\Omega}\left\|\boldsymbol{\varphi}_{\mathbf{u}}\right\|_{L^{4}\left(0, T ; \mathbf{W}^{2,4}(\Omega)\right)}\left(\int_{0}^{T}\left\|\mathbf{y}_{\mathbf{u}}-\overline{\mathbf{y}}\right\|_{\mathbf{L}^{2}(\Omega)}^{2}\left\|\nabla \mathbf{y}_{\mathbf{u}}-\nabla \overline{\mathbf{y}}\right\|_{\mathbf{L}^{2}(\Omega)}^{2} \mathrm{~d} t\right)^{1 / 4} \\
& \leq \sqrt{2} C_{\Omega}\left\|\boldsymbol{\varphi}_{\mathbf{u}}\right\|_{L^{4}\left(0, T ; \mathbf{W}^{2,4}(\Omega)\right)}\left\|\mathbf{y}_{\mathbf{u}}-\overline{\mathbf{y}}\right\|_{L^{\infty}\left(0, T ; \mathbf{H}_{0}^{1}(\Omega)\right)}^{1 / 2}\left\|\mathbf{y}_{\mathbf{u}}-\overline{\mathbf{y}}\right\|_{\mathbf{L}^{2}\left(\Omega_{T}\right)}^{1 / 2} \\
& \leq \sqrt{2} C_{\Omega} C_{\boldsymbol{\alpha}, \boldsymbol{\beta}}^{2}\left\|\mathbf{y}_{\mathbf{u}}-\overline{\mathbf{y}}\right\|_{L^{\infty}\left(0, T ; \mathbf{H}_{0}^{1}(\Omega)\right)}^{1 / 2}\left\|\mathbf{y}_{\mathbf{u}}-\overline{\mathbf{y}}\right\|_{\mathbf{L}^{2}\left(\Omega_{T}\right)}^{1 / 2} \leq \hat{C}_{\boldsymbol{\alpha}, \boldsymbol{\beta}}\left\|\mathbf{y}_{\mathbf{u}}-\overline{\mathbf{y}}\right\|_{\mathbf{L}^{2}\left(\Omega_{T}\right)}^{1 / 2},
\end{aligned}
$$

where

$$
\hat{C}_{\boldsymbol{\alpha}, \boldsymbol{\beta}}=\sqrt{2} C_{\Omega} C_{\boldsymbol{\alpha}, \boldsymbol{\beta}}^{2} \max \left\{\left\|\mathbf{y}_{\mathbf{u}}-\overline{\mathbf{y}}\right\|_{L^{\infty}\left(0, T ; \mathbf{H}_{0}^{1}(\Omega)\right)}^{1 / 2}: \mathbf{u} \in \mathcal{U}_{a d}\right\}
$$

Finally, we get the estimate for the third term with (3.29)

$$
\begin{aligned}
\left\|\left(\nabla \mathbf{y}_{\mathbf{u}}-\overline{\mathbf{y}}\right)^{T} \boldsymbol{\varphi}_{\mathbf{u}}\right\|_{\mathbf{L}^{2}\left(\Omega_{T}\right)} & \leq\left\|\boldsymbol{\varphi}_{\mathbf{u}}\right\|_{\mathbf{L} \infty\left(\Omega_{T}\right)}\left\|\nabla \mathbf{y}_{\mathbf{u}}-\nabla \overline{\mathbf{y}}\right\|_{\mathbf{L}^{2}\left(\Omega_{T}\right)} \\
& \leq \tilde{C}_{\boldsymbol{\alpha}, \boldsymbol{\beta}}\left\|\mathbf{y}_{\mathbf{u}}-\overline{\mathbf{y}}\right\|_{\mathbf{L}^{2}\left(\Omega_{T}\right)}^{1 / 2},
\end{aligned}
$$

where

$$
\tilde{C}_{\boldsymbol{\alpha}, \boldsymbol{\beta}}=\max \left\{\left(\sqrt{L_{2}} L_{\infty} \tilde{M}\|\mathbf{u}-\overline{\mathbf{u}}\|_{\mathbf{L}^{2}\left(\Omega_{T}\right)}^{\frac{2}{\bar{p}}}+D_{\boldsymbol{\alpha}, \boldsymbol{\beta}}\right)\left\|\boldsymbol{\varphi}_{\mathbf{u}}\right\|_{\mathbf{L}^{\infty}\left(\Omega_{T}\right)}\|\mathbf{u}-\overline{\mathbf{u}}\|_{\mathbf{L}^{2}\left(\Omega_{T}\right)}^{1 / 2}: \mathbf{u} \in \mathcal{U}_{a d}\right\}
$$

Now, (3.36)-(3.38) imply (3.31). 
Remark 3.12. It is clear that the estimate (3.31) is still valid, even in the three dimensional case provided that we minimize the functional (1.3). First, we note that in this case (3.34) takes the form:

$$
\left\{\begin{aligned}
-\overline{\boldsymbol{\varphi}}_{t}-\nu \Delta \overline{\boldsymbol{\varphi}}-(\overline{\mathbf{y}} \cdot \nabla) \overline{\boldsymbol{\varphi}}+(\nabla \overline{\mathbf{y}})^{T} \overline{\boldsymbol{\varphi}}+\nabla \pi & \\
& =\left\|\overline{\mathbf{y}}-\mathbf{y}_{d}\right\|_{\mathbf{L}^{4}(\Omega)}^{4}\left|\overline{\mathbf{y}}-\mathbf{y}_{d}\right|^{2}\left(\overline{\mathbf{y}}-\mathbf{y}_{d}\right) \text { in } \Omega_{T} \\
\operatorname{div} \bar{\varphi} & =0 \text { in } \Omega_{T}, \bar{\varphi}(T)=0 \text { in } \Omega, \overline{\boldsymbol{\varphi}}=0 \text { on } \Sigma_{T}
\end{aligned}\right.
$$

However, due to $L^{\infty}\left(\Omega_{T}\right)$ regularity of $\overline{\mathbf{y}}$, the terms on the right can be handled easily (see also [6]). It remains to check the various terms involving $\bar{\varphi}$, and in particular (3.37). However, note that (3.37), holds with different constants, since $\left\|\mathbf{y}_{\mathbf{u}}-\overline{\mathbf{y}}\right\|_{\mathbf{L}^{4}\left(\Omega_{T}\right)} \leq\left\|\mathbf{y}_{\mathbf{u}}-\overline{\mathbf{y}}\right\|_{L^{\infty}\left(\Omega_{T}\right)}^{1 / 2}\left\|\mathbf{y}_{\mathbf{u}}-\overline{\mathbf{y}}\right\|_{\mathbf{L}^{2}\left(\Omega_{T}\right)}^{1 / 2}$. The rest of the proof remains the same, since we have not used any other result involving embeddings.

Lemma 3.13. For every $\rho>0$ there exists $\varepsilon>0$ such that

$$
\left|\left[J^{\prime \prime}(\mathbf{u})-J^{\prime \prime}(\overline{\mathbf{u}})\right](\mathbf{u}-\overline{\mathbf{u}})^{2}\right| \leq \rho\left\|\mathbf{z}_{\mathbf{u}-\overline{\mathbf{u}}}\right\|_{\mathbf{L}^{2}\left(\Omega_{T}\right)}^{2}
$$

$\forall \mathbf{u} \in \mathcal{U}_{a d}$ with $\|\mathbf{u}-\overline{\mathbf{u}}\|_{\mathbf{L}^{2}\left(\Omega_{T}\right)}<\varepsilon$.

Proof. Let us take $\mathbf{u} \in \mathcal{U}_{\text {ad }}$ and denote $\mathbf{z}_{\mathbf{u}-\overline{\mathbf{u}}}=G^{\prime}(\overline{\mathbf{u}})(\mathbf{u}-\overline{\mathbf{u}})$ and $\mathbf{z}_{\mathbf{u}, \mathbf{u}-\overline{\mathbf{u}}}=G^{\prime}(\mathbf{u})(\mathbf{u}-\overline{\mathbf{u}})$. Then, according to $(3.2)$, we have

$$
\begin{aligned}
& J^{\prime \prime}(\mathbf{u})(\mathbf{u}-\overline{\mathbf{u}})^{2}-J^{\prime \prime}(\overline{\mathbf{u}})(\mathbf{u}-\overline{\mathbf{u}})^{2}=\int_{\Omega_{T}}\left[\left|\mathbf{z}_{\mathbf{u}, \mathbf{u}-\overline{\mathbf{u}}}\right|^{2}-\left|\mathbf{z}_{\mathbf{u}-\overline{\mathbf{u}}}\right|^{2}\right] \mathrm{d} x \mathrm{~d} t \\
& +2 \int_{\Omega_{T}}\left[\left(\mathbf{z}_{\mathbf{u}, \mathbf{u}-\overline{\mathbf{u}}} \cdot \nabla\right) \mathbf{z}_{\mathbf{u}, \mathbf{u}-\overline{\mathbf{u}}} \boldsymbol{\varphi}_{\mathbf{u}}-\left(\mathbf{z}_{\mathbf{u}-\overline{\mathbf{u}}} \cdot \nabla\right) \mathbf{z}_{\mathbf{u}-\overline{\mathbf{u}} \bar{\varphi}]} d x \mathrm{~d} t=I_{1}+I_{2} .\right.
\end{aligned}
$$

Let us estimate $I_{1}$ and $I_{2}$. For the estimate of $I_{1}$ we use (3.23), (3.22) and (2.6) as follows

$$
\begin{aligned}
& \left|I_{1}\right| \leq\left(\left\|\mathbf{z}_{\mathbf{u}, \mathbf{u}-\overline{\mathbf{u}}}\right\|_{\mathbf{L}^{2}\left(\Omega_{T}\right)}+\left\|\mathbf{z}_{\mathbf{u}-\overline{\mathbf{u}}}\right\|_{\mathbf{L}^{2}\left(\Omega_{T}\right)}\right)\left\|\mathbf{z}_{\mathbf{u}, \mathbf{u}-\overline{\mathbf{u}}}-\mathbf{z}_{\mathbf{u}-\overline{\mathbf{u}}}\right\|_{\mathbf{L}^{2}\left(\Omega_{T}\right)} \\
& \leq\left(2+\sqrt{T} K_{\boldsymbol{\alpha}, \boldsymbol{\beta}}\left\|\mathbf{y}_{\mathbf{u}}-\overline{\mathbf{y}}\right\|_{\mathbf{L}^{\infty}\left(\Omega_{T}\right)}\right)\left\|\mathbf{z}_{\mathbf{u}-\overline{\mathbf{u}}}\right\|_{\mathbf{L}^{2}\left(\Omega_{T}\right)} K_{\boldsymbol{\alpha}, \boldsymbol{\beta}}\left\|\mathbf{y}_{\mathbf{u}}-\overline{\mathbf{y}}\right\|_{\mathbf{L}^{\infty}\left(\Omega_{T}\right)}\left\|\mathbf{z}_{\mathbf{u}-\overline{\mathbf{u}}}\right\|_{\mathbf{L}^{2}\left(\Omega_{T}\right)}
\end{aligned}
$$

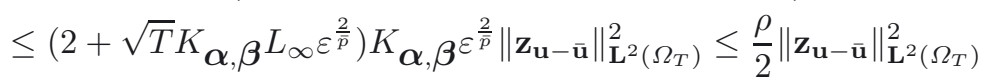

where $\varepsilon$ is chosen so that

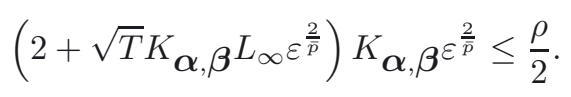

For the estimate of $I_{2}$ we write

$$
\begin{aligned}
& I_{2}=2 \int_{\Omega_{T}}\left[\left(\mathbf{z}_{\mathbf{u}, \mathbf{u}-\overline{\mathbf{u}}}-\mathbf{z}_{\mathbf{u}-\overline{\mathbf{u}}}\right) \cdot \nabla\right] \mathbf{z}_{\mathbf{u}, \mathbf{u}-\overline{\mathbf{u}}} \boldsymbol{\varphi}_{\mathbf{u}} \mathrm{d} x \mathrm{~d} t \\
& +2 \int_{\Omega_{T}}\left(\mathbf{z}_{\mathbf{u}-\overline{\mathbf{u}}} \cdot \nabla\right)\left(\mathbf{z}_{\mathbf{u}, \mathbf{u}-\overline{\mathbf{u}}}-\mathbf{z}_{\mathbf{u}-\overline{\mathbf{u}}}\right) \boldsymbol{\varphi}_{\mathbf{u}} \mathrm{d} x \mathrm{~d} t \\
& +2 \int_{\Omega_{T}}\left(\mathbf{z}_{\mathbf{u}-\overline{\mathbf{u}}} \cdot \nabla\right) \mathbf{z}_{\mathbf{u}-\overline{\mathbf{u}}}\left(\boldsymbol{\varphi}_{\mathbf{u}}-\bar{\varphi}\right) \mathrm{d} x \mathrm{~d} t=I_{21}+I_{22}+I_{23} .
\end{aligned}
$$


Now, using again (3.22) and (3.24) we have

$$
\begin{aligned}
& \left|I_{21}\right| \leq 2 \int_{0}^{T}\left|c\left(\mathbf{z}_{\mathbf{u}, \mathbf{u}-\overline{\mathbf{u}}}-\mathbf{z}_{\mathbf{u}-\overline{\mathbf{u}}}, \mathbf{z}_{\mathbf{u}, \mathbf{u}-\overline{\mathbf{u}}}, \boldsymbol{\varphi}_{\mathbf{u}}\right)\right| \mathrm{d} t=2 \int_{0}^{T}\left|c\left(\mathbf{z}_{\mathbf{u}, \mathbf{u}-\overline{\mathbf{u}}}-\mathbf{z}_{\mathbf{u}-\overline{\mathbf{u}}}, \boldsymbol{\varphi}_{\mathbf{u}}, \mathbf{z}_{\mathbf{u}, \mathbf{u}-\overline{\mathbf{u}}}\right)\right| \mathrm{d} t \\
& \leq 2 \int_{0}^{T}\left\|\mathbf{z}_{\mathbf{u}, \mathbf{u}-\overline{\mathbf{u}}}-\mathbf{z}_{\mathbf{u}-\overline{\mathbf{u}}}\right\|_{\mathbf{L}^{2}(\Omega)}\left\|\nabla \boldsymbol{\varphi}_{\mathbf{u}}\right\|_{\mathbf{L}^{\infty}(\Omega)}\left\|\mathbf{z}_{\mathbf{u}, \mathbf{u}-\overline{\mathbf{u}}}\right\|_{\mathbf{L}^{2}(\Omega)} \mathrm{d} t \\
& \leq 2 C_{\Omega}\left\|\mathbf{z}_{\mathbf{u}, \mathbf{u}-\overline{\mathbf{u}}}-\mathbf{z}_{\mathbf{u}-\overline{\mathbf{u}}}\right\|_{C\left([0, T] ; \mathbf{L}^{2}(\Omega)\right)} \int_{0}^{T}\left\|\boldsymbol{\varphi}_{\mathbf{u}}\right\|_{\mathbf{W}^{2, \bar{p}}(\Omega)}\left\|\mathbf{z}_{\mathbf{u}, \mathbf{u}-\overline{\mathbf{u}}}\right\|_{\mathbf{L}^{2}(\Omega)} \mathrm{d} t \\
& \leq 2 C_{\Omega} K_{\boldsymbol{\alpha}, \boldsymbol{\beta}}\left\|\mathbf{y}_{\mathbf{u}}-\overline{\mathbf{y}}\right\|_{\mathbf{L}^{\infty}\left(\Omega_{T}\right)}\left\|\mathbf{z}_{\mathbf{u}-\overline{\mathbf{u}}}\right\|_{\mathbf{L}^{2}\left(\Omega_{T}\right)}\left\|\boldsymbol{\varphi}_{\mathbf{u}}\right\|_{L^{2}\left(0, T ; \mathbf{W}^{2, \bar{p}}(\Omega)\right.}\left\|\mathbf{z}_{\mathbf{u}, \mathbf{u}-\overline{\mathbf{u}}}\right\|_{\mathbf{L}^{2}\left(\Omega_{T}\right)}
\end{aligned}
$$

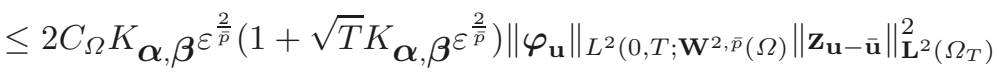

$$
\begin{aligned}
& \leq \frac{\rho}{6}\left\|\mathbf{z}_{\mathbf{u}-\overline{\mathbf{u}}}\right\|_{\mathbf{L}^{2}\left(\Omega_{T}\right)}^{2}
\end{aligned}
$$

with

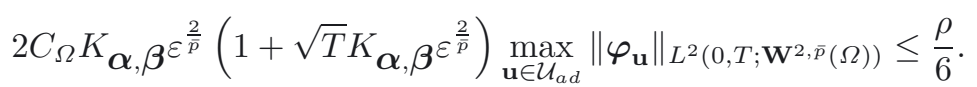

For $I_{22}$ we observe that

$$
I_{22} \leq 2 \int_{0}^{T}\left|c\left(\mathbf{z}_{\mathbf{u}, \mathbf{u}-\overline{\mathbf{u}}}, \mathbf{z}_{\mathbf{u}, \mathbf{u}-\overline{\mathbf{u}}}-\mathbf{z}_{\mathbf{u}-\overline{\mathbf{u}}}, \boldsymbol{\varphi}_{\mathbf{u}}\right)\right| \mathrm{d} t=2 \int_{0}^{T}\left|c\left(\mathbf{z}_{\mathbf{u}, \mathbf{u}-\overline{\mathbf{u}}}, \boldsymbol{\varphi}_{\mathbf{u}}, \mathbf{z}_{\mathbf{u}, \mathbf{u}-\overline{\mathbf{u}}}-\mathbf{z}_{\mathbf{u}-\overline{\mathbf{u}}}\right)\right| \mathrm{d} t .
$$

Hence, we can proceed as in (3.45) and obtain the same estimate. Finally, to estimate $I_{23}$ we use (3.31) and (3.28). Moreover, we choose $\varepsilon$ as in Lemma 3.8, so that (3.21) holds. Thus, we get

$$
\begin{aligned}
& \left|I_{23}\right| \leq 2 \int_{0}^{T}\left|c\left(\mathbf{z}_{\mathbf{u}-\overline{\mathbf{u}}}, \mathbf{z}_{\mathbf{u}-\overline{\mathbf{u}}}, \boldsymbol{\varphi}_{\mathbf{u}}-\overline{\boldsymbol{\varphi}}\right)\right| \mathrm{d} t=2 \int_{0}^{T}\left|c\left(\mathbf{z}_{\mathbf{u}-\overline{\mathbf{u}}}, \boldsymbol{\varphi}_{\mathbf{u}}-\overline{\boldsymbol{\varphi}}, \mathbf{z}_{\mathbf{u}-\overline{\mathbf{u}}}\right)\right| \mathrm{d} t \\
& \leq 2 \int_{0}^{T}\left\|\boldsymbol{\varphi}_{\mathbf{u}}-\bar{\varphi}\right\|_{\mathbf{H}_{0}^{1}(\Omega)}\left\|\mathbf{z}_{\mathbf{u}-\overline{\mathbf{u}}}\right\|_{\mathbf{L}^{4}(\Omega)}^{2} \mathrm{~d} t \\
& \leq 4\left\|\boldsymbol{\varphi}_{\mathbf{u}}-\bar{\varphi}\right\|_{L^{\infty}\left(0, T ; \mathbf{H}_{0}^{1}(\Omega)\right)} \int_{0}^{T}\left\|\mathbf{z}_{\mathbf{u}-\overline{\mathbf{u}}}\right\|_{\mathbf{L}^{2}(\Omega)}\left\|\nabla \mathbf{z}_{\mathbf{u}-\overline{\mathbf{u}}}\right\|_{\mathbf{L}^{2}(\Omega)} \mathrm{d} t \\
& \leq 4 E_{\boldsymbol{\alpha}, \boldsymbol{\beta}}\left\|\mathbf{y}_{\mathbf{u}}-\overline{\mathbf{y}}\right\|_{\mathbf{L}^{2}\left(\Omega_{T}\right)}^{1 / 2}\left\|\mathbf{z}_{\mathbf{u}-\overline{\mathbf{u}}}\right\|_{\mathbf{L}^{2}\left(\Omega_{T}\right)}\left\|\nabla \mathbf{z}_{\mathbf{u}-\overline{\mathbf{u}}}\right\|_{\mathbf{L}^{2}\left(\Omega_{T}\right)} \\
& \leq 4 E_{\boldsymbol{\alpha}, \boldsymbol{\beta}^{D}} \boldsymbol{\alpha}, \boldsymbol{\beta}_{\boldsymbol{\beta}}\|\mathbf{u}-\overline{\mathbf{u}}\|_{\mathbf{L}^{2}\left(\Omega_{T}\right)}^{1 / 2}\left\|\mathbf{y}_{\mathbf{u}}-\overline{\mathbf{y}}\right\|_{\mathbf{L}^{2}\left(\Omega_{T}\right)}\left\|\mathbf{z}_{\mathbf{u}-\overline{\mathbf{u}}}\right\|_{\mathbf{L}^{2}\left(\Omega_{T}\right)}
\end{aligned}
$$

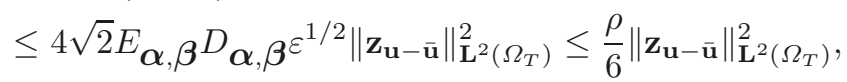

with $\varepsilon$ satisfying

$$
4 \sqrt{2} E_{\boldsymbol{\alpha}, \boldsymbol{\beta}^{D}} \boldsymbol{\alpha}, \boldsymbol{\beta}^{\varepsilon^{1 / 2}} \leq \frac{\rho}{6}
$$

Therefore, if $\varepsilon$ is chosen according to Lemma 3.8 and satisfying (3.42), (3.45) and (3.47), then the inequality (3.39) follows from (3.40), (3.41), (3.43), (3.44) (which is also an estimate for $I_{22}$ ) and (3.46). 
Remark 3.14. In the three dimensional case, the estimate (3.39) is still valid. Recall, that the only change concerns (3.46), where using the inequality $\|\cdot\|_{L^{4}} \leq C\|\cdot\|_{L^{2}}^{1 / 4}\|\cdot\|_{H^{1}}^{3 / 4}$, Hölder's inequality, (3.21), and (3.28), we obtain:

$$
\begin{aligned}
& \left|I_{23}\right| \leq 2 \int_{0}^{T}\left|c\left(\mathbf{z}_{\mathbf{u}-\overline{\mathbf{u}}}, \mathbf{z}_{\mathbf{u}-\overline{\mathbf{u}}}, \boldsymbol{\varphi}_{\mathbf{u}}-\overline{\boldsymbol{\varphi}}\right)\right| \mathrm{d} t=2 \int_{0}^{T}\left|c\left(\mathbf{z}_{\mathbf{u}-\overline{\mathbf{u}}}, \boldsymbol{\varphi}_{\mathbf{u}}-\overline{\boldsymbol{\varphi}}, \mathbf{z}_{\mathbf{u}-\overline{\mathbf{u}}}\right)\right| \mathrm{d} t \\
& \leq 2 \int_{0}^{T}\left\|\boldsymbol{\varphi}_{\mathbf{u}}-\overline{\boldsymbol{\varphi}}\right\|_{\mathbf{H}_{0}^{1}(\Omega)}\left\|\mathbf{z}_{\mathbf{u}-\overline{\mathbf{u}}}\right\|_{\mathbf{L}^{4}(\Omega)}^{2} \mathrm{~d} t \\
& \leq 4\left\|\boldsymbol{\varphi}_{\mathbf{u}}-\bar{\varphi}\right\|_{L^{\infty}\left(0, T ; \mathbf{H}_{0}^{1}(\Omega)\right)} \int_{0}^{T}\left\|\mathbf{z}_{\mathbf{u}-\overline{\mathbf{u}}}\right\|_{\mathbf{L}^{2}(\Omega)}^{1 / 2}\left\|\nabla \mathbf{z}_{\mathbf{u}-\overline{\mathbf{u}}}\right\|_{\mathbf{L}^{2}(\Omega)}^{3 / 2} \mathrm{~d} t \\
& \leq 4 E_{\boldsymbol{\alpha}, \boldsymbol{\beta}}\left\|\mathbf{y}_{\mathbf{u}}-\overline{\mathbf{y}}\right\|_{\mathbf{L}^{2}\left(\Omega_{T}\right)}^{1 / 2}\left\|\mathbf{z}_{\mathbf{u}-\overline{\mathbf{u}}}\right\|_{\mathbf{L}^{2}\left(\Omega_{T}\right)}^{1 / 2}\left\|\nabla \mathbf{z}_{\mathbf{u}-\overline{\mathbf{u}}}\right\|_{\mathbf{L}^{2}\left(\Omega_{T}\right)}^{3 / 2} \\
& \leq 4 E_{\boldsymbol{\alpha}, \boldsymbol{\beta}^{D}} \boldsymbol{\alpha}, \boldsymbol{\beta}_{\boldsymbol{\beta}}\|\mathbf{u}-\overline{\mathbf{u}}\|_{\mathbf{L}^{2}\left(\Omega_{T}\right)}^{3 / 4}\left\|\mathbf{y}_{\mathbf{u}}-\overline{\mathbf{y}}\right\|_{\mathbf{L}^{2}\left(\Omega_{T}\right)}^{3 / 2}\left\|\mathbf{z}_{\mathbf{u}-\overline{\mathbf{u}}}\right\|_{\mathbf{L}^{2}\left(\Omega_{T}\right)}^{1 / 2} \\
& \leq 2^{3 / 2} 4 E_{\boldsymbol{\alpha}, \boldsymbol{\beta}^{D}} \boldsymbol{\alpha}, \boldsymbol{\beta}^{\varepsilon^{3 / 4}}\left\|\mathbf{z}_{\mathbf{u}-\overline{\mathbf{u}}}\right\|_{\mathbf{L}^{2}\left(\Omega_{T}\right)}^{2} \leq \frac{\rho}{6}\left\|\mathbf{z}_{\mathbf{u}-\overline{\mathbf{u}}}\right\|_{\mathbf{L}^{2}\left(\Omega_{T}\right)}^{2},
\end{aligned}
$$

with $\varepsilon$ satisfying

$$
42^{3 / 2} E_{\boldsymbol{\alpha}, \boldsymbol{\beta}^{D}} \boldsymbol{\alpha}, \boldsymbol{\beta}^{\varepsilon^{3 / 4}} \leq \frac{\rho}{6}
$$

Lemma 3.15. There exists a constant $F_{\boldsymbol{\alpha}, \boldsymbol{\beta}}$ such that the following inequality holds

$$
\left|J^{\prime \prime}(\mathbf{u}) \mathbf{v}^{2}\right| \leq F_{\boldsymbol{\alpha}, \boldsymbol{\beta}}\|\mathbf{v}\|_{\mathbf{L}^{2}\left(\Omega_{T}\right)}\left\|\mathbf{z}_{\mathbf{v}}\right\|_{\mathbf{L}^{2}\left(\Omega_{T}\right)} \quad \forall \mathbf{u} \in \mathcal{U}_{a d} \text { and } \forall \mathbf{v} \in \mathbf{L}^{2}\left(\Omega_{T}\right),
$$

where $\mathbf{z}_{\mathbf{v}}=G^{\prime}(\mathbf{u}) \mathbf{v}$ is the solution of $(2.4)$.

Proof. From Theorem 2.5 with $\bar{p}=2$, we deduce the existence of a constant such that

$$
\left\|\mathbf{z}_{\mathbf{v}}\right\|_{\mathbf{L}^{2}\left(\Omega_{T}\right)}+\left\|\nabla \mathbf{z}_{\mathbf{v}}\right\|_{\mathbf{L}^{2}\left(\Omega_{T}\right)} \leq C_{2}\|\mathbf{v}\|_{\mathbf{L}^{2}\left(\Omega_{T}\right)} \forall \mathbf{u} \in \mathcal{U}_{a d} \text { and } \forall \mathbf{v} \in \mathbf{L}^{2}\left(\Omega_{T}\right) .
$$

From this inequality and (3.2) we obtain

$\left|J^{\prime \prime}(\mathbf{u}) \mathbf{v}^{2}\right| \leq\left\|\mathbf{z}_{\mathbf{v}}\right\|_{\mathbf{L}^{2}\left(\Omega_{T}\right)}^{2}+2\left\|\boldsymbol{\varphi}_{\mathbf{u}}\right\|_{\mathbf{L}^{\infty}\left(\Omega_{T}\right)}\left\|\mathbf{z}_{\mathbf{v}}\right\|_{\mathbf{L}^{2}\left(\Omega_{T}\right)}\left\|\nabla \mathbf{z}_{\mathbf{v}}\right\|_{\mathbf{L}^{2}\left(\Omega_{T}\right)} \leq C_{2}\left(1+2\left\|\boldsymbol{\varphi}_{\mathbf{u}}\right\|_{\mathbf{L}^{\infty}\left(\Omega_{T}\right)}\right)\|\mathbf{v}\|_{\mathbf{L}^{2}\left(\Omega_{T}\right)}\left\|\mathbf{z}_{\mathbf{v}}\right\|_{\mathbf{L}^{2}\left(\Omega_{T}\right)}$, which implies (3.48) with

$$
F_{\boldsymbol{\alpha}, \boldsymbol{\beta}}=\max \left\{C_{2}\left(1+2\left\|\boldsymbol{\varphi}_{\mathbf{u}}\right\|_{\mathbf{L}^{\infty}\left(\Omega_{T}\right)}\right): \mathbf{u} \in \mathcal{U}_{a d}\right\}
$$

Proof of Theorem 3.6. Let $\varepsilon>0$ to be fixed later, and $\mathbf{u} \in \mathcal{U}_{\text {ad }}$ with $\|\mathbf{u}-\overline{\mathbf{u}}\|_{\mathbf{L}^{2}\left(\Omega_{T}\right)}<\varepsilon$. We will distinguish two cases.

Case I: $\mathbf{u}-\overline{\mathbf{u}} \notin C_{\overline{\mathbf{u}}}^{\varrho}$. In this case we assume that

$$
\varepsilon<\min \left\{\frac{1}{C_{2}}, \frac{2 \varrho}{F_{\boldsymbol{\alpha}, \boldsymbol{\beta}}}, \frac{1-\frac{1}{\sqrt{2}}}{\bar{M} \sqrt{T}}\right\},
$$

where $\bar{M}, C_{2}$ and $F_{\boldsymbol{\alpha}, \boldsymbol{\beta}}$ are given in Lemma 3.7, (3.49) and Lemma 3.15, respectively. 
Since $\mathbf{u} \in \mathcal{U}_{a d}, \mathbf{u}-\overline{\mathbf{u}}$ obviously satisfies the sign conditions (3.9)-(3.10). Therefore, from the definition of $C_{\overline{\mathbf{u}}}^{\varrho}$, we deduce that $J^{\prime}(\overline{\mathbf{u}})(\mathbf{u}-\overline{\mathbf{u}})>\varrho\left\|\mathbf{z}_{\mathbf{u}-\overline{\mathbf{u}}}\right\|_{\mathbf{L}^{2}\left(\Omega_{T}\right)}$. Then, making a Taylor expansion and using this inequality and (3.48) we get

$$
\begin{aligned}
J(\mathbf{u}) & =J(\overline{\mathbf{u}})+J^{\prime}(\overline{\mathbf{u}})(\mathbf{u}-\overline{\mathbf{u}})+\frac{1}{2} J^{\prime \prime}(\overline{\mathbf{u}}+\theta(\mathbf{u}-\overline{\mathbf{u}}))(\mathbf{u}-\overline{\mathbf{u}})^{2} \\
& >J(\overline{\mathbf{u}})+\varrho\left\|\mathbf{z}_{\mathbf{u}-\overline{\mathbf{u}}}\right\|_{\mathbf{L}^{2}\left(\Omega_{T}\right)}-\frac{1}{2 F_{\boldsymbol{\alpha}, \boldsymbol{\beta}}}\|\mathbf{u}-\overline{\mathbf{u}}\|_{\mathbf{L}^{2}\left(\Omega_{T}\right)}\left\|\mathbf{z}_{\mathbf{u}-\overline{\mathbf{u}}}\right\|_{\mathbf{L}^{2}\left(\Omega_{T}\right)} \\
& =J(\overline{\mathbf{u}})+\left(\frac{2 \varrho-F_{\boldsymbol{\alpha}, \boldsymbol{\beta}^{\varepsilon}}}{2}\right)\left\|\mathbf{z}_{\mathbf{u}-\overline{\mathbf{u}}}\right\|_{\mathbf{L}^{2}\left(\Omega_{T}\right)} \geq J(\overline{\mathbf{u}})+\left(\frac{2 \varrho-F_{\boldsymbol{\alpha}, \boldsymbol{\beta}^{\varepsilon}}}{2}\right) \| \mathbf{z}_{\mathbf{u}-\overline{\mathbf{u}} \|_{\mathbf{L}^{2}\left(\Omega_{T}\right)}} .
\end{aligned}
$$

In the last inequality we have used (3.49) and (3.50) to infer

$$
\left\|\mathbf{z}_{\mathbf{u}-\overline{\mathbf{u}}}\right\|_{\mathbf{L}^{2}\left(\Omega_{T}\right)} \leq C_{2} \varepsilon \leq 1 .
$$

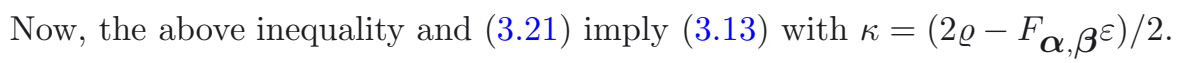

Case II: $\mathbf{u}-\overline{\mathbf{u}} \in C_{\overline{\mathbf{u}}}^{\varrho}$. Let us take $\rho=\delta / 2$ in Lemma 3.13 and select $\varepsilon>0$ such that (3.39) holds. Additionally, we assume that $\varepsilon$ satisfies (3.50). We make a Taylor expansion again

$$
J(\mathbf{u})=J(\overline{\mathbf{u}})+J^{\prime}(\overline{\mathbf{u}})(\mathbf{u}-\overline{\mathbf{u}})+\frac{1}{2} J^{\prime \prime}(\overline{\mathbf{u}})(\mathbf{u}-\overline{\mathbf{u}})^{2}+\frac{1}{2 \theta^{2}}\left[J^{\prime \prime}\left(\mathbf{u}_{\theta}\right)-J^{\prime \prime}(\overline{\mathbf{u}})\right]\left(\mathbf{u}_{\theta}-\overline{\mathbf{u}}\right)^{2},
$$

where $\mathbf{u}_{\theta}=\overline{\mathbf{u}}+\theta(\mathbf{u}-\overline{\mathbf{u}})$. Now, from (3.1) and (3.6) we deduce that $J^{\prime}(\overline{\mathbf{u}})(\mathbf{u}-\overline{\mathbf{u}}) \geq 0$. Thus, using (3.12), (3.39), the identity $\mathbf{z}_{\mathbf{u}_{\theta}-\overline{\mathbf{u}}}=\theta \mathbf{z}_{\mathbf{u}-\overline{\mathbf{u}}}$ and that $0<\theta<1$ we get

$$
J(\mathbf{u}) \geq J(\overline{\mathbf{u}})+\frac{\delta}{2}\left\|\mathbf{z}_{\mathbf{u}-\overline{\mathbf{u}}}\right\|_{\mathbf{L}^{2}\left(\Omega_{T}\right)}^{2}-\frac{\delta}{4 \theta^{2}}\left\|\mathbf{z}_{\mathbf{u}_{\theta}-\overline{\mathbf{u}}}\right\|_{\mathbf{L}^{2}\left(\Omega_{T}\right)}^{2}=J(\overline{\mathbf{u}})+\frac{\delta}{4}\left\|\mathbf{z}_{\mathbf{u}-\overline{\mathbf{u}}}\right\|_{\mathbf{L}^{2}\left(\Omega_{T}\right)}^{2} .
$$

This inequality and (3.21) imply (3.13) with $\kappa=\delta / 4$.

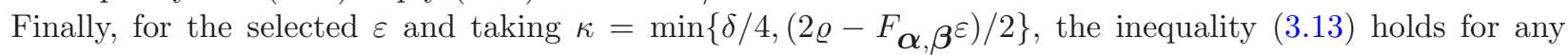
case.

\section{Numerical approximation of the CONTROL PROBlem}

In this section we assume that $\Omega$ is a convex set and we consider a family of triangulations $\left\{\mathcal{K}_{h}\right\}_{h>0}$ of $\bar{\Omega}$, defined in the standard way. To each element $K \in \mathcal{K}_{h}$, we associate two parameters $h_{K}$ and $\varrho_{K}$, where $h_{K}$ denotes the diameter of the set $K$ and $\varrho_{K}$ is the diameter of the largest ball contained in $K$. Define the size of the mesh by $h=\max _{K \in \mathcal{K}_{h}} h_{K}$. We also assume that the standard regularity assumptions on the triangulation:

(i) There exist two positive constants $\varrho_{\mathcal{K}}$ and $\delta_{\mathcal{K}}$ such that $\frac{h_{K}}{\varrho_{K}} \leq \varrho_{\mathcal{K}}$ and $\frac{h}{h_{K}} \leq \delta_{\mathcal{K}} \forall K \in \mathcal{K}_{h}$ and $\forall h>0$.

(ii) Define $\bar{\Omega}_{h}=\cup_{K \in \mathcal{K}_{h}} K$, and let $\Omega_{h}$ and $\Gamma_{h}$ denote its interior and its boundary, respectively. We assume that the vertices of $\mathcal{K}_{h}$ placed on the boundary $\Gamma_{h}$ are points of $\Gamma$.

Since $\Omega$ is convex, from the last assumption we have that $\Omega_{h}$ is also convex. Moreover, we know that

$$
\left|\Omega \backslash \Omega_{h}\right| \leq C h^{2}
$$

see, for instance ([27], estimate (5.2.19)).

On the mesh $\mathcal{K}_{h}$ we consider two finite dimensional spaces $\mathbf{Z}_{h} \subset \mathbf{H}_{0}^{1}(\Omega)$ and $Q_{h} \subset L_{0}^{2}(\Omega)$ formed by piecewise polynomials in $\Omega_{h}$ and vanishing in $\Omega \backslash \Omega_{h}$. We make the following assumptions on these spaces. 
(A1) If $\mathbf{z} \in \mathbf{H}^{1+l}(\Omega) \cap \mathbf{H}_{0}^{1}(\Omega)$, then

$$
\inf _{\mathbf{z}_{h} \in \mathbf{Z}_{h}}\left\|\mathbf{z}-\mathbf{z}_{h}\right\|_{\mathbf{H}^{s}\left(\Omega_{h}\right)} \leq C h^{l+1-s}\|\mathbf{z}\|_{\mathbf{H}^{1+l}(\Omega)}, \text { for } 0 \leq l \leq 1 \text { and } s=0,1 .
$$

(A2) If $q \in H^{l}(\Omega) \cap L_{0}^{2}(\Omega)$, then

$$
\inf _{q_{h} \in Q_{h}}\left\|q-q_{h}\right\|_{L^{2}\left(\Omega_{h}\right)} \leq C h\|q\|_{H^{1}(\Omega)} .
$$

(A3) The subspaces $\mathbf{Z}_{h}$ and $Q_{h}$ satisfy the inf-sup condition: $\exists c>0$ such that

$$
\inf _{q_{h} \in Q_{h}} \sup _{\mathbf{z}_{h} \in \mathbf{Z}_{h}} \frac{b\left(\mathbf{z}_{h}, q_{h}\right)}{\left\|\mathbf{z}_{h}\right\|_{\mathbf{H}^{1}\left(\Omega_{h}\right)}\left\|q_{h}\right\|_{L^{2}\left(\Omega_{h}\right)}} \geq c,
$$

where $b: \mathbf{H}^{1}(\Omega) \times L^{2}(\Omega) \longrightarrow \mathbb{R}$ is defined by

$$
b(\mathbf{z}, q)=\int_{\Omega} q(x) \operatorname{div} \mathbf{z}(x) \mathrm{d} x .
$$

These assumptions are satisfied by the usual finite elements considered in the discretization of Navier-Stokes equations: "Taylor-Hood", P1-Bubble finite element, and some others (see [15], Chap. 2). In addition, we assume that classical inverse estimates on uniformly regular meshes for $\mathbf{Z}_{h}$ hold.

We also consider a subspace $\mathbf{Y}_{h}$ of $\mathbf{Z}_{h}$ defined by

$$
\mathbf{Y}_{h}=\left\{\mathbf{y}_{h} \in \mathbf{Z}_{h}: b\left(\mathbf{y}_{h}, q_{h}\right)=0 \forall q_{h} \in Q_{h}\right\} .
$$

We proceed now with the discretization in time. Let us consider a grid of points $0=t_{0}<t_{1}<\ldots<t_{N_{\tau}}=T$. We denote $\tau_{n}=t_{n}-t_{n-1}$. We assume that we construct a quasi-uniform in time partition, i.e.,

$$
\exists \varrho_{0} \in(0,1] \text { s.t. } \tau=\max _{1 \leq n \leq N_{\tau}} \tau_{n} \text { satisfies } \varrho_{0} \tau \leq \tau_{n} \quad \forall 1 \leq n \leq N_{\tau} \text { and } \forall \tau>0 .
$$

Given a triangulation $\mathcal{K}_{h}$ of $\Omega$ and a grid of points $\left\{t_{n}\right\}_{n=0}^{N_{\tau}}$ of $[0, T]$, we set $\sigma=(\tau, h)$. Finally, we consider the following spaces

$$
\begin{aligned}
& \mathcal{Y}_{\sigma}=\left\{\mathbf{y}_{\sigma} \in L^{2}\left(0, T ; \mathbf{Y}_{h}\right): \mathbf{y}_{\left.\sigma\right|_{\left(t_{n-1}, t_{n}\right)}} \in \mathbf{Y}_{h} \text { for } 1 \leq n \leq N_{\tau}\right\} \\
& \mathcal{Q}_{\sigma}=\left\{q_{\sigma} \in L^{2}\left(0, T ; Q_{h}\right): q_{\left.\sigma\right|_{\left(t_{n-1}, t_{n}\right)}} \in Q_{h} \text { for } 1 \leq n \leq N_{\tau}\right\}
\end{aligned}
$$

We have that the functions of $\mathcal{Y}_{\sigma}$ and $\mathcal{Q}_{\sigma}$ are piecewise constant in time. The elements of $\mathcal{Y}_{\sigma}$ can be written in the form

$$
\mathbf{y}_{\sigma}=\sum_{n=1}^{N_{\tau}} \mathbf{y}_{n, h} \chi_{n}, \quad \text { with } \mathbf{y}_{n, h} \in \mathbf{Y}_{h},
$$

where $\chi_{n}$ is the characteristic function of $\left(t_{n-1}, t_{n}\right)$. For every discrete state $\mathbf{y}_{\sigma}$ we will fix $\mathbf{y}_{\sigma}\left(t_{n}\right)=\mathbf{y}_{n, h}$, so that $\mathbf{y}_{\sigma}$ is continuous on the left. In particular, we have $\mathbf{y}_{\sigma}(T)=\mathbf{y}_{\sigma}\left(t_{N_{\tau}}\right)=y_{N_{\tau}, h}$.

\subsection{The discrete state equation}

To define the discrete control problem we have to consider the numerical discretization of the state equation (1.1) or equivalently (2.1). We achieve this goal by using a discontinuous time-stepping Galerkin method, with piecewise constants in time and conforming finite element spaces in space. For any $\mathbf{u} \in L^{2}\left(0, T ; \mathbf{L}^{2}(\Omega)\right)$ the discrete state equation is given by:

$$
\left\{\begin{array}{l}
\text { For } n=1, \ldots, N_{\tau}, \\
\left(\frac{\mathbf{y}_{n, h}-\mathbf{y}_{n-1, h}}{\tau_{n}}, \mathbf{w}_{h}\right)+a\left(\mathbf{y}_{n, h}, \mathbf{w}_{h}\right)+c\left(\mathbf{y}_{n, h}, \mathbf{y}_{n, h}, \mathbf{w}_{h}\right) \\
=\left(\mathbf{f}_{n}+\mathbf{u}_{n}, \mathbf{w}_{h}\right) \quad \forall \mathbf{w}_{h} \in \mathbf{Y}_{h}, \\
\mathbf{y}_{0, h}=\mathbf{y}_{0 h},
\end{array}\right.
$$


where

$$
\begin{aligned}
& \left(\mathbf{f}_{n}, \mathbf{w}_{h}\right)=\frac{1}{\tau_{n}} \int_{t_{n-1}}^{t_{n}}\left(\mathbf{f}(t), \mathbf{w}_{h}\right) \mathrm{d} t,\left(\mathbf{u}_{n}, \mathbf{w}_{h}\right)=\frac{1}{\tau_{n}} \int_{t_{n-1}}^{t_{n}}\left(\mathbf{u}(t), \mathbf{w}_{h}\right) \mathrm{d} t \\
& \mathbf{y}_{0 h} \in \mathbf{Y}_{h} \text { with }\left\|\mathbf{y}_{0}-\mathbf{y}_{0 h}\right\|_{\mathbf{L}^{2}\left(\Omega_{h}\right)} \leq C h, \text { and }\left\|\mathbf{y}_{0 h}\right\|_{\mathbf{H}^{1}\left(\Omega_{h}\right)} \leq C
\end{aligned}
$$

It well-known that the discrete equation (4.7) has at least one solution. Concerning uniqueness and error estimates under the prescribed regularity assumptions, the following results was proven in ([4], Thm. 4.7).

Theorem 4.1. Given $\mathbf{u} \in L^{2}\left(0, T ; \mathbf{L}^{2}(\Omega)\right)$, let us denote the solution of $(2.1)$ by $\mathbf{y} \in \mathbf{H}^{2,1}\left(\Omega_{T}\right) \cap C([0, T] ; \mathbf{Y})$, and let $\mathbf{y}_{\sigma} \in \mathcal{Y}_{\sigma}$ be any solution of (4.7). Then, there exists a constant $C>0$ independent of $\mathbf{u}, \mathbf{y}$ and $\sigma$ such that

$$
\begin{aligned}
& \max _{1 \leq n \leq N_{\tau}}\left\|\mathbf{y}\left(t_{n}\right)-\mathbf{y}_{\sigma}\left(t_{n}\right)\right\|+\left\|\mathbf{y}-\mathbf{y}_{\sigma}\right\|_{L^{2}\left(0, T ; \mathbf{H}^{1}\left(\Omega_{h}\right)\right)} \\
& \leq C\left\{\frac{\tau}{h}\left\|\mathbf{y}^{\prime}\right\|_{L^{2}\left(0, T ; \mathbf{L}^{2}(\Omega)\right)}+h\|\mathbf{y}\|_{L^{2}\left(0, T ; \mathbf{H}^{2}(\Omega)\right)}+h\left\|\mathbf{y}_{0}\right\|_{\mathbf{H}^{1}(\Omega)}\right\} \\
& \left\|\mathbf{y}-\mathbf{y}_{\sigma}\right\|_{L^{\infty}\left(0, T ; \mathbf{L}^{2}\left(\Omega_{h}\right)\right)} \leq C\left\{\left(\frac{\tau}{h}+\sqrt{\tau}\right)\left\|\mathbf{y}^{\prime}\right\|_{L^{2}\left(0, T ; \mathbf{L}^{2}(\Omega)\right)}\right. \\
& \left.+h\|\mathbf{y}\|_{L^{2}\left(0, T ; \mathbf{H}^{2}(\Omega)\right)}+h\left\|\mathbf{y}_{0}\right\|_{\mathbf{H}^{1}(\Omega)}\right\}
\end{aligned}
$$

Moreover, if there exists a constant $C_{0}>0$ such that $\tau \leq C_{0} h^{2}$ for every $\sigma=(\tau, h)$, then $\left\{\mathbf{y}_{\sigma}\right\}_{\sigma}$ is bounded in $L^{\infty}\left(0, T ; \mathbf{H}^{1}\left(\Omega_{h}\right)\right)$ and there exists $\sigma_{0}>0$ such that (4.7) has a unique solution for every $|\sigma| \leq \sigma_{0}$.

By using the above results, and a suitable duality argument the following estimate was proved in [5].

Theorem 4.2. Under the notation and assumptions of Theorem 4.1 and including the hypothesis $\tau \leq C_{0} h^{2}$ for some constant $C_{0}$, the following estimate holds:

$$
\left\|\mathbf{y}-\mathbf{y}_{\sigma}\right\|_{L^{2}\left(0, T ; \mathbf{L}^{2}\left(\Omega_{h}\right)\right)} \leq C h^{2},
$$

where $C$ is independent of $\sigma$. Moreover, the same constant $C$ can be taken for every $\mathbf{u} \in \mathcal{U}_{a d}$.

\subsection{The discrete adjoint-state equation}

In this section, the assumptions $\tau \leq C_{0} h^{2}$ and $|\sigma| \leq \sigma_{0}$ will be assumed, with $\sigma_{0}$ as introduced in Theorem 4.1. Then, associated to the discrete state equation (4.7), the cost functional $J$ is approximated by $J_{\sigma}: \mathbf{L}^{2}\left(\Omega_{T}\right) \longrightarrow$ $\mathbb{R}$, given by:

$$
J_{\sigma}(\mathbf{u})=\frac{1}{2} \int_{0}^{T} \int_{\Omega_{h}}\left|\mathbf{y}_{\sigma}(\mathbf{u})-\mathbf{y}_{d}\right|^{2} \mathrm{~d} x \mathrm{~d} t .
$$

The functional $J_{\sigma}$ is of class $C^{\infty}$, and we have a first expression of its derivative as follows

$$
J_{\sigma}^{\prime}(\mathbf{u}) \mathbf{v}=\int_{0}^{T} \int_{\Omega_{h}}\left(\mathbf{y}_{\sigma}(\mathbf{u})-\mathbf{y}_{d}\right) \mathbf{z}_{\sigma} \mathrm{d} x \mathrm{~d} t,
$$

where $\mathbf{z}_{\sigma}$ is the solution of the linearized equation

$$
\left\{\begin{array}{l}
\text { For } n=1, \ldots, N_{\tau}, \\
\left(\frac{\mathbf{z}_{n, h}-\mathbf{z}_{n-1, h}}{\tau_{n}}, \mathbf{w}_{h}\right)+a\left(\mathbf{z}_{n, h}, \mathbf{w}_{h}\right)+c\left(\mathbf{z}_{n, h}, \mathbf{y}_{n, h}, \mathbf{w}_{h}\right) \\
+c\left(\mathbf{y}_{n, h}, \mathbf{z}_{n, h}, \mathbf{w}_{h}\right)=\frac{1}{\tau_{n}} \int_{t_{n-1}}^{t_{n}}\left(\mathbf{v}(t), \mathbf{w}_{h}\right) \mathrm{d} t \forall \mathbf{w}_{h} \in \mathbf{Y}_{h}, \\
\mathbf{z}_{0, h}=0,
\end{array}\right.
$$


where $\mathbf{y}_{\sigma}=\sum_{n=1}^{N_{\tau}} \mathbf{y}_{n, h} \chi_{n}$; see [4] for details. By using the adjoint state equation

$$
\left\{\begin{array}{l}
\text { for } n=N_{\tau}, \ldots, 1, \text { and } \forall \mathbf{w}_{h} \in \mathbf{Y}_{h} \\
\left(\frac{\boldsymbol{\varphi}_{n, h}-\boldsymbol{\varphi}_{n+1, h}}{\tau_{n}}, \mathbf{w}_{h}\right)+a\left(\boldsymbol{\varphi}_{n, h}, \mathbf{w}_{h}\right)+c\left(\mathbf{w}_{h}, \mathbf{y}_{n, h}, \boldsymbol{\varphi}_{n, h}\right) \\
+c\left(\mathbf{y}_{n, h}, \mathbf{w}_{h}, \boldsymbol{\varphi}_{n, h}\right)=\frac{1}{\tau_{n}} \int_{t_{n-1}}^{t_{n}}\left(\mathbf{y}_{n, h}-\mathbf{y}_{d}(t), \mathbf{w}_{h}\right) \mathrm{d} t \\
\boldsymbol{\varphi}_{N_{\tau}+1, h}=0,
\end{array}\right.
$$

the derivative of $J_{\sigma}$ can be expressed as

$$
J_{\sigma}^{\prime}(\mathbf{u}) \mathbf{v}=\int_{0}^{T} \int_{\Omega_{h}} \varphi_{\sigma} \mathbf{v} \mathrm{d} x \mathrm{~d} t
$$

Observe that in the above system (4.14), first we compute $\varphi_{N_{\tau}, h}$ from $\varphi_{N_{\tau}+1, h}=0$ and then we descend in $n$ until $n=1$. Unlike the discrete states $\mathbf{y}_{\sigma}$, we will set for the discrete adjoint states $\boldsymbol{\varphi}_{\sigma}\left(t_{n-1}\right)=\boldsymbol{\varphi}_{n, h}$ for every $1 \leq n \leq N_{\tau}$.

\subsection{The discrete optimal control problem}

As in Section 4.2, the hypotheses $\tau \leq C_{0} h^{2}$ and $|\sigma| \leq \sigma_{0}$ will be assumed throughout this section. Here the goal is to prove the convergence of the discrete optimal controls to a continuous one and to derive some error estimates for the optimal states. To this end, we first define the discrete control space. Let us denote

$$
\mathbf{U}_{h}=\left\{\mathbf{u}_{h} \in \mathbf{L}^{2}\left(\Omega_{h}\right): \mathbf{u}_{h \mid K} \equiv \mathbf{u}_{K} \in \mathbb{R}^{2} \quad \forall K \in \mathcal{K}_{h}\right\}
$$

and

$$
\mathcal{U}_{\sigma}=\left\{\mathbf{u}_{\sigma} \in L^{2}\left(0, T ; \mathbf{U}_{h}\right):\left.\mathbf{u}_{\sigma}\right|_{\left(t_{n-1}, t_{n}\right)} \in \mathbf{U}_{h}, \text { for } 1 \leq n \leq N_{\tau}\right\}
$$

Hence, every element of $\mathcal{U}_{\sigma}$ can be written in the form

$$
\mathbf{u}_{\sigma}=\sum_{n=1}^{N_{\tau}} \sum_{K \in \mathcal{K}_{h}} \mathbf{u}_{n, K} \chi_{n} \chi_{K}
$$

where $\chi_{n}$ and $\chi_{K}$ denote the characteristic functions of $\left(t_{n-1}, t_{n}\right)$ and $K$, respectively. Now, the discrete control problem is formulated as follows

$$
\left(\mathrm{P}_{\sigma}\right) \quad\left\{\begin{array}{l}
\min J_{\sigma}\left(\mathbf{u}_{\sigma}\right) \\
\mathbf{u}_{\sigma} \in \mathcal{U}_{\sigma, a d}
\end{array}\right.
$$

where

$$
J_{\sigma}\left(\mathbf{u}_{\sigma}\right)=\frac{1}{2} \int_{0}^{T} \int_{\Omega_{h}}\left|\mathbf{y}_{\sigma}\left(\mathbf{u}_{\sigma}\right)-\mathbf{y}_{d}\right|^{2} \mathrm{~d} x \mathrm{~d} t \quad \text { and } \quad \mathcal{U}_{\sigma, a d}=\mathcal{U}_{a d} \cap \mathcal{U}_{\sigma}
$$


Theorem 4.3. For every $\sigma$, the control problem $\left(\mathrm{P}_{\sigma}\right)$ has at least one solution. Moreover, if $\overline{\mathbf{u}}_{\sigma}$ is a local minimum of $\left(\mathrm{P}_{\sigma}\right)$, then there exist $\overline{\mathbf{y}}_{\sigma}, \overline{\boldsymbol{\varphi}}_{\sigma} \in \mathcal{Y}_{\sigma}$ such that

$$
\begin{aligned}
& \left\{\begin{array}{l}
\text { For } n=1, \ldots, N_{\tau}, \\
\left(\frac{\overline{\mathbf{y}}_{n, h}-\overline{\mathbf{y}}_{n-1, h}}{\tau_{n}}, \mathbf{w}_{h}\right)+a\left(\overline{\mathbf{y}}_{n, h}, \mathbf{w}_{h}\right)+c\left(\overline{\mathbf{y}}_{n, h}, \overline{\mathbf{y}}_{n, h}, \mathbf{w}_{h}\right) \\
=\left(\mathbf{f}_{n}+\overline{\mathbf{u}}_{n}, \mathbf{w}_{h}\right) \quad \forall \mathbf{w}_{h} \in \mathbf{Y}_{h}, \\
\overline{\mathbf{y}}_{0, h}=\mathbf{y}_{0 h},
\end{array}\right. \\
& \left\{\begin{array}{l}
\text { for } n=N_{\tau}, \ldots, 1, \quad \text { and } \quad \forall \mathbf{w}_{h} \in \mathbf{Y}_{h}, \\
\left(\frac{\overline{\boldsymbol{\varphi}}_{n, h}-\overline{\boldsymbol{\varphi}}_{n+1, h}}{\tau_{n}}, \mathbf{w}_{h}\right)+a\left(\overline{\boldsymbol{\varphi}}_{n, h}, \mathbf{w}_{h}\right)+c\left(\mathbf{w}_{h}, \overline{\mathbf{y}}_{n, h}, \overline{\boldsymbol{\varphi}}_{n, h}\right) \\
+c\left(\overline{\mathbf{y}}_{n, h}, \mathbf{w}_{h}, \overline{\boldsymbol{\varphi}}_{n, h}\right)=\frac{1}{\tau_{n}} \int_{t_{n-1}}^{t_{n}}\left(\overline{\mathbf{y}}_{n, h}-\mathbf{y}_{d}(t), \mathbf{w}_{h}\right) \mathrm{d} t, \\
\overline{\boldsymbol{\varphi}}_{N_{\tau}+1, h}=0,
\end{array}\right. \\
& \int_{0}^{T} \int_{\Omega_{h}} \overline{\boldsymbol{\varphi}}_{\sigma}(x, t)\left(\mathbf{u}_{\sigma}(x, t)-\overline{\mathbf{u}}_{\sigma}(x, t)\right) \mathrm{d} x \mathrm{~d} t \geq 0 \quad \forall \mathbf{u}_{\sigma} \in \mathcal{U}_{\sigma, a d},
\end{aligned}
$$

where

$$
\left(\mathbf{f}_{n}+\overline{\mathbf{u}}_{n}, \mathbf{w}_{h}\right)=\frac{1}{\tau_{n}} \int_{t_{n-1}}^{t_{n}}\left(\mathbf{f}(t)+\overline{\mathbf{u}}_{\sigma}(t), \mathbf{w}_{h}\right) \mathrm{d} t=\frac{1}{\tau_{n}} \int_{t_{n-1}}^{t_{n}}\left(\mathbf{f}(t), \mathbf{w}_{h}\right) \mathrm{d} t+\left(\overline{\mathbf{u}}_{n}, \mathbf{w}_{h}\right) .
$$

The existence of a solution is an immediate consequence of the continuity of $J_{\sigma}$, the compactness of $\mathcal{U}_{\sigma, a d}$ in $\mathcal{U}_{\sigma}$ and the fact that $\mathcal{U}_{\sigma, a d} \neq \emptyset$. The optimality system follows from (4.7), (4.14) and the expression for the derivative of $J_{\sigma}^{\prime}$ given in Section 4.2. The inequality (4.17) says that $J_{\sigma}^{\prime}\left(\overline{\mathbf{u}}_{\sigma}\right)\left(\mathbf{u}_{\sigma}-\overline{\mathbf{u}}_{\sigma}\right) \geq 0 \forall \mathbf{u}_{\sigma} \in \mathcal{U}_{\sigma, a d}$.

Taking into account the definition of $\mathcal{U}_{\sigma},(4.17)$ can be written in the form

$$
\sum_{n=1}^{N_{\tau}} \sum_{K \in \mathcal{K}_{h}}\left(\int_{t_{n-1}}^{t_{n}} \int_{K} \overline{\boldsymbol{\varphi}}_{\sigma}(x, t) \mathrm{d} x \mathrm{~d} t\right)\left(\mathbf{u}_{n, K}-\overline{\mathbf{u}}_{n, K}\right) \geq 0 \quad \forall \mathbf{u}_{n, K} \in[\boldsymbol{\alpha}, \boldsymbol{\beta}],
$$

or equivalently

$$
\left(\int_{t_{n-1}}^{t_{n}} \int_{K} \overline{\boldsymbol{\varphi}}_{\sigma}(x, t) \mathrm{d} x \mathrm{~d} t\right)\left(\boldsymbol{\xi}-\overline{\mathbf{u}}_{n, K}\right) \geq 0 \quad \forall \boldsymbol{\xi} \in[\boldsymbol{\alpha}, \boldsymbol{\beta}], 1 \leq n \leq N_{\tau}, K \in \mathcal{K}_{h} .
$$

Using this fact, we obtain the discrete version of (3.7)

$$
\left\{\begin{array} { c l } 
{ \overline { u } _ { n , K , j } = \alpha _ { j } } & { \Rightarrow I _ { n , K , j } \geq 0 , } \\
{ \overline { u } _ { n , K , j } = \beta _ { j } } & { \Rightarrow I _ { n , K , j } \leq 0 , } \\
{ \alpha _ { j } < \overline { u } _ { n , K , j } < \beta _ { j } } & { \Rightarrow I _ { n , K , j } = 0 , }
\end{array} \text { and } \left\{\begin{array}{rl}
I_{n, K, j}>0 \Rightarrow \bar{u}_{n, K, j}=\alpha_{j}, \\
I_{n, K, j}<0 \Rightarrow \bar{u}_{n, K, j}=\beta_{j},
\end{array}\right.\right.
$$

where

$$
I_{n, K, j}=\int_{t_{n-1}}^{t_{n}} \int_{K} \bar{\varphi}_{\sigma, j}(x, t) \mathrm{d} x \mathrm{~d} t
$$

and $\bar{\varphi}_{\sigma}=\left(\bar{\varphi}_{\sigma, 1}, \bar{\varphi}_{\sigma, 2}\right)$.

The next theorem proves the convergence of the solutions of $\left(\mathrm{P}_{\sigma}\right)$ towards solutions of $(\mathrm{P})$.

Theorem 4.4. Let $\left\{\overline{\mathbf{u}}_{\sigma}\right\}_{\sigma}$ be a sequence of solutions of problems $\left(\mathrm{P}_{\sigma}\right)$ and let $\left\{\overline{\mathbf{y}}_{\sigma}\right\}_{\sigma}$ be the associated discrete states. Then, if $\overline{\mathbf{u}}$ is the weak limit in $\mathbf{L}^{2}\left(\Omega_{T}\right)$ of $\left\{\overline{\mathbf{u}}_{\sigma}\right\}_{\sigma}$ as $\sigma \rightarrow 0$, then $\overline{\mathbf{u}}$ is a solution of $(\mathrm{P})$. Moreover, $\left\{\overline{\mathbf{y}}_{\sigma}\right\}_{\sigma}$ converges strongly to $\overline{\mathbf{y}}$ in $\mathbf{L}^{2}\left(\Omega_{T}\right)$, where $\overline{\mathbf{y}}$ is the continuous state associated with $\overline{\mathbf{u}}$. In addition, if $\overline{\mathbf{u}}$ is a bang-bang control, then $\overline{\mathbf{u}}_{\sigma} \rightarrow \overline{\mathbf{u}}$ as $\sigma \rightarrow 0$ strongly in $\mathbf{L}^{p}\left(\Omega_{T}\right)$ for every $1 \leq p<+\infty$. 
Proof. If $\overline{\mathbf{u}}_{\sigma} \rightarrow \overline{\mathbf{u}}$ in $\mathbf{L}^{2}\left(\Omega_{T}\right)$ as $\sigma \rightarrow 0$, then Corollary 2.4 implies that $\mathbf{y}_{\overline{\mathbf{u}}_{\sigma}} \rightarrow \overline{\mathbf{y}}$ strongly in $\mathbf{L}^{2}\left(\Omega_{T}\right)$, where $\mathbf{y}_{\overline{\mathbf{u}}_{\sigma}}$ denotes the continuous state associated with $\overline{\mathbf{u}}_{\sigma}: \mathbf{y}_{\overline{\mathbf{u}}_{\sigma}}=G\left(\overline{\mathbf{u}}_{\sigma}\right)$. Moreover, Theorem 4.2 implies that $\overline{\mathbf{y}}_{\sigma}-\mathbf{y}_{\overline{\mathbf{u}}_{\sigma}} \rightarrow 0$ strongly in $\mathbf{L}^{2}\left(\Omega_{T}\right)$. Hence, $\overline{\mathbf{y}}_{\sigma} \rightarrow \overline{\mathbf{y}}$ strongly in $\mathbf{L}^{2}\left(\Omega_{T}\right)$.

Let us prove that $\overline{\mathbf{u}}$ is a solution of $(\mathrm{P})$. Let $\mathbf{u} \in \mathcal{U}_{a d}$ and take $\mathbf{u}_{\sigma}$ as the $\mathbf{L}^{2}\left(\Omega_{T}\right)$ projection of $\mathbf{u}$ on $\mathcal{U}_{\sigma}$ :

$$
\mathbf{u}_{n, K}=\frac{1}{\tau_{n}|K|} \int_{t_{n-1}}^{t_{n}} \int_{K} \mathbf{u}(x, t) \mathrm{d} x \mathrm{~d} t, \quad 1 \leq n \leq N_{\tau}, K \in \mathcal{K} .
$$

It is well-known that the projections converge strongly: $\mathbf{u}_{\sigma} \rightarrow \mathbf{u}$ in $\mathbf{L}^{2}\left(\Omega_{T}\right)$. Arguing as above, we also obtain that $\mathbf{y}_{\sigma}\left(\mathbf{u}_{\sigma}\right) \rightarrow \mathbf{y}_{\mathbf{u}}$ strongly in $\mathbf{L}^{2}\left(\Omega_{T}\right)$. Moreover, from the expression in (4.20) it follows that $\mathbf{u}_{\sigma}(x, t) \in[\boldsymbol{\alpha}, \boldsymbol{\beta}]$, therefore $\mathbf{u}_{\sigma} \in \mathcal{U}_{\sigma, a d}$. Hence, we have

$$
J(\overline{\mathbf{u}})=\lim _{\sigma \rightarrow 0} J_{\sigma}\left(\overline{\mathbf{u}}_{\sigma}\right) \leq \lim _{\sigma \rightarrow 0} J_{\sigma}\left(\mathbf{u}_{\sigma}\right)=J(\mathbf{u}) .
$$

Since $\mathbf{u} \in \mathcal{U}_{\text {ad }}$ is arbitrary, we deduce that $\overline{\mathbf{u}}$ is a solution of $(\mathrm{P})$.

Now, we prove the strong convergence of the controls. For $j=1,2$ let us consider the sets

$$
\Omega_{T, \boldsymbol{\alpha}, j}=\left\{(x, t) \in \Omega_{T}: \bar{u}_{j}(x, t)=\alpha_{j}\right\} \text { and } \Omega_{T, \boldsymbol{\beta}, j}=\left\{(x, t) \in \Omega_{T}: \bar{u}_{j}(x, t)=\beta_{j}\right\} .
$$

Let us extend $\bar{u}_{\sigma, j}$ to $\Omega_{T}$ with the only restriction that it is measurable and $\alpha_{j} \leq \bar{u}_{\sigma, j}(x, t) \leq \beta_{j}$ in $\Omega_{T}$. Since $\overline{\mathbf{u}}$ is a bang-bang control, we have that $\left|\Omega_{T, \boldsymbol{\alpha}, j}\right|+\left|\Omega_{T, \boldsymbol{\beta}, j}\right|=\left|\Omega_{T}\right|$. Using that $\bar{u}_{\sigma, j} \rightarrow \bar{u}_{j}$ in $L^{1}\left(\Omega_{T}\right)$ as $|\sigma| \rightarrow 0$, we get

$$
\int_{\Omega_{T}}\left|\bar{u}_{\sigma, j}-\bar{u}_{j}\right| \mathrm{d} x \mathrm{~d} t=\int_{\Omega_{T, \boldsymbol{\alpha}, j}}\left(\bar{u}_{\sigma, j}-\bar{u}_{j}(x, t)\right) \mathrm{d} x \mathrm{~d} t+\int_{\Omega_{T, \boldsymbol{\beta}_{, j}}}\left(\bar{u}_{j}(x, t)-\bar{u}_{\sigma, j}\right) \mathrm{d} x \mathrm{~d} t \rightarrow 0 \quad \text { as }|\sigma| \rightarrow 0 .
$$

Hence $\bar{u}_{\sigma, j} \rightarrow \bar{u}_{j}$ in $L^{1}\left(\Omega_{T}\right)$. Since $\left\{\bar{u}_{\sigma, j}\right\}_{\sigma}$ is bounded in $L^{\infty}\left(\Omega_{T}\right)$ we deduce that $\bar{u}_{\sigma, j} \rightarrow \bar{u}_{j}$ strongly in $L^{p}\left(\Omega_{T}\right)$ for every $1 \leq p<\infty$.

Remark 4.5. Using (4.12) and an inverse inequality, we can prove that the sequence $\left\{\overline{\mathbf{y}}_{\sigma}\right\}_{\sigma}$ is bounded in $\mathbf{L}^{\infty}\left(\Omega_{T}\right)$, hence $\overline{\mathbf{y}}_{\sigma} \rightarrow \overline{\mathbf{y}}$ strongly in $\mathbf{L}^{p}\left(\Omega_{T}\right)$ for every $1 \leq p<+\infty$.

Now we have the following converse result.

Theorem 4.6. Let $\overline{\mathbf{u}}$ be a strict local minimum of $(\mathrm{P})$. We also assume that $\overline{\mathbf{u}}$ is a bang-bang control. Then, there exist $\varepsilon>0, \sigma_{0}>0$ and a sequence $\left\{\overline{\mathbf{u}}_{\sigma}\right\}_{|\sigma| \leq \sigma_{0}}$, such that each $\overline{\mathbf{u}}_{\sigma}$ is a local solution of $\left(\mathrm{P}_{\sigma}\right)$, and the following properties hold

1. $J_{\sigma}\left(\overline{\mathbf{u}}_{\sigma}\right) \leq J_{\sigma}\left(\mathbf{u}_{\sigma}\right) \forall \mathbf{u}_{\sigma} \in \mathcal{U}_{\sigma, a d} \cap \bar{B}_{\varepsilon}(\overline{\mathbf{u}}), \bar{B}_{\varepsilon}(\overline{\mathbf{u}})$ denoting the $\mathbf{L}^{2}\left(\Omega_{T}\right)$ ball,

2. $\overline{\mathbf{u}}_{\sigma} \rightarrow \overline{\mathbf{u}}$ strongly in $\mathbf{L}^{p}\left(\Omega_{T}\right)$ for every $1 \leq p<+\infty$.

Proof. Let us take $\varepsilon>0$ such that $J(\overline{\mathbf{u}})<J(\mathbf{u})$ for every $\mathbf{u} \in \mathcal{U}_{a d} \backslash\{\overline{\mathbf{u}}\}$ with $\|\mathbf{u}-\overline{\mathbf{u}}\|_{\mathbf{L}^{2}\left(\Omega_{T}\right)} \leq \varepsilon$. Let us define the set $\mathcal{U}_{a d, \varepsilon}=\mathcal{U}_{a d} \cap \bar{B}_{\varepsilon}(\overline{\mathbf{u}})$, where $\bar{B}_{\varepsilon}(\overline{\mathbf{u}})$ is the $\mathbf{L}^{2}\left(\Omega_{T}\right)$ ball centered at $\overline{\mathbf{u}}$ and radius $\varepsilon$. Then, $\overline{\mathbf{u}}$ is the unique solution of the problem

$$
\left(\mathrm{P}_{\varepsilon}\right)\left\{\begin{array}{l}
\min J(\mathbf{u}) \\
\mathbf{u} \in \mathcal{U}_{a d, \varepsilon}
\end{array}\right.
$$

Now, we consider the discrete problem

$$
\left(\mathrm{P}_{\sigma, \varepsilon}\right) \quad\left\{\begin{array}{l}
\min J_{\sigma}\left(\mathbf{u}_{\sigma}\right) \\
\mathbf{u}_{\sigma} \in \mathcal{U}_{\sigma, a d, \varepsilon}
\end{array}\right.
$$

where $\mathcal{U}_{\sigma, a d, \varepsilon}=\mathcal{U}_{a d, \varepsilon} \cap \mathcal{U}_{\sigma}$. Let $\hat{\mathbf{u}}_{\sigma}$ be the $\mathbf{L}^{2}\left(\Omega_{T}\right)$ projection of $\overline{\mathbf{u}}$ on $\mathcal{U}_{\sigma}$; see (4.20). We know that $\hat{\mathbf{u}}_{\sigma} \rightarrow \overline{\mathbf{u}}$ strongly in $\mathbf{L}^{2}\left(\Omega_{T}\right)$. Therefore, there exists $\sigma_{0}$ such that $\hat{\mathbf{u}}_{\sigma} \in \mathcal{U}_{\sigma, a d, \varepsilon}$ for every $|\sigma| \leq \sigma_{0}$. Hence, $\mathcal{U}_{\sigma, a d, \varepsilon}$ is non 
empty for $|\sigma| \leq \sigma_{0}$, and $\left(\mathrm{P}_{\sigma, \varepsilon}\right)$ has at least one solution $\overline{\mathbf{u}}_{\sigma}$. Since $\left\{\overline{\mathbf{u}}_{\sigma}\right\}_{|\sigma| \leq \sigma_{0}} \subset \mathcal{U}_{a d, \varepsilon}$, we can take a subsequence, denoted in the same way, such that $\overline{\mathbf{u}}_{\sigma} \stackrel{*}{\rightarrow} \tilde{\mathbf{u}}$ in $\mathbf{L}^{\infty}\left(\Omega_{T}\right)$ with $\tilde{\mathbf{u}} \in \mathcal{U}_{a d, \varepsilon}$. Let us denote by $\left\{\overline{\mathbf{y}}_{\sigma}\right\}_{|\sigma| \leq \sigma_{0}}$ the discrete states associated with the controls $\left\{\overline{\mathbf{u}}_{\sigma}\right\}_{|\sigma| \leq \sigma_{0}}$ and let $\tilde{\mathbf{y}}=G(\tilde{\mathbf{u}})$ be the continuous state corresponding to $\tilde{\mathbf{u}}$. Arguing as in the proof of Theorem 4.4, we get that $\overline{\mathbf{y}}_{\sigma} \rightarrow \tilde{\mathbf{y}}$ strongly in $\mathbf{L}^{2}\left(\Omega_{T}\right)$. Then, we have

$$
J(\tilde{\mathbf{u}})=\lim _{\sigma \rightarrow 0} J_{\sigma}\left(\overline{\mathbf{u}}_{\sigma}\right) \leq \lim _{\sigma \rightarrow 0} J_{\sigma}\left(\hat{\mathbf{u}}_{\sigma}\right)=J(\overline{\mathbf{u}}) .
$$

Hence, $\tilde{\mathbf{u}}$ is a solution of $\left(\mathrm{P}_{\varepsilon}\right)$. But, $\overline{\mathbf{u}}$ is the unique solution of $\left(\mathrm{P}_{\varepsilon}\right)$, consequently $\tilde{\mathbf{u}}=\overline{\mathbf{u}}$. Finally, the strong convergence $\overline{\mathbf{u}}_{\sigma} \rightarrow \overline{\mathbf{u}}$ is established as in the proof of Theorem 4.4.

We conclude this section by proving some error estimates for the optimal states.

Theorem 4.7. Let $\overline{\mathbf{u}}$ be a local solution of $(\mathrm{P})$ with associated state $\overline{\mathbf{y}}$. We assume that (3.12) holds. Let $\left\{\overline{\mathbf{u}}_{\sigma}\right\}_{\sigma}$ be a sequence of local minima of problems $\left(\mathrm{P}_{\sigma}\right)$ such that $J_{\sigma}\left(\overline{\mathbf{u}}_{\sigma}\right) \leq J_{\sigma}\left(\mathbf{u}_{\sigma}\right) \forall \mathbf{u}_{\sigma} \in \mathcal{U}_{\sigma, a d} \cap \bar{B}_{\varepsilon}(\overline{\mathbf{u}})$, where $\bar{B}_{\varepsilon}(\overline{\mathbf{u}})$ denotes a $\mathbf{L}^{2}\left(\Omega_{T}\right)$ ball, and $\overline{\mathbf{u}}_{\sigma} \rightarrow \overline{\mathbf{u}}$ in $\mathbf{L}^{2}\left(\Omega_{T}\right)$. Let $\left\{\overline{\mathbf{y}}_{\sigma}\right\}_{\sigma}$ be the corresponding discrete states. Then, there exists a constant $C>0$ independent of $\sigma$ such that

$$
\lim _{|\sigma| \rightarrow 0} \frac{1}{\sqrt{|\sigma|}}\left\|\overline{\mathbf{y}}_{\sigma}-\overline{\mathbf{y}}\right\|_{\mathbf{L}^{2}\left(\Omega_{T}\right)}=0 .
$$

Proof. Here, we recall that $\tau \leq C_{0} h^{2}$ has been assumed, hence $|\sigma| \sim h$ as $\sigma \rightarrow 0$. Along the proof, $C$ will be used to define any constant independent of $\sigma$. Let $\varepsilon>0$ be given such that (3.13) holds. Since $\overline{\mathbf{u}}_{\sigma} \rightarrow \overline{\mathbf{u}}$, there exists $\sigma_{0}>0$ such that $\left\|\overline{\mathbf{u}}_{\sigma}-\overline{\mathbf{u}}\right\|_{\mathbf{L}^{2}\left(\Omega_{T}\right)}<\varepsilon$ for every $|\sigma| \leq \sigma_{0}$. Then, (3.13) implies that

$$
\frac{\kappa}{2}\left\|\mathbf{y}_{\overline{\mathbf{u}}_{\sigma}}-\overline{\mathbf{y}}\right\|_{\mathbf{L}^{2}\left(\Omega_{T}\right)}^{2} \leq J\left(\overline{\mathbf{u}}_{\sigma}\right)-J(\overline{\mathbf{u}}),
$$

where $\mathbf{y}_{\overline{\mathbf{u}}_{\sigma}}=G\left(\overline{\mathbf{u}}_{\sigma}\right)$ is the continuous solution of (1.1) associated to $\overline{\mathbf{u}}_{\sigma}$. To prove (4.21) we use (4.12) as follows

$$
\left\|\overline{\mathbf{y}}_{\sigma}-\overline{\mathbf{y}}\right\|_{\mathbf{L}^{2}\left(\Omega_{T}\right)} \leq\left\|\overline{\mathbf{y}}_{\sigma}-\mathbf{y}_{\overline{\mathbf{u}}_{\sigma}}\right\|_{\mathbf{L}^{2}\left(\Omega_{T}\right)}+\left\|\mathbf{y}_{\overline{\mathbf{u}}_{\sigma}}-\overline{\mathbf{y}}\right\|_{\mathbf{L}^{2}\left(\Omega_{T}\right)} \leq C h^{2}+\left\|\mathbf{y}_{\overline{\mathbf{u}}_{\sigma}}-\overline{\mathbf{y}}\right\|_{\mathbf{L}^{2}\left(\Omega_{T}\right)} .
$$

We will provide an estimate for the right hand side of (4.22), which together (4.23) will lead to (4.21). Let us denote by $\mathbf{u}_{\sigma}$ the $\mathbf{L}^{2}\left(\Omega_{T}\right)$ projection of $\overline{\mathbf{u}}$ on $\mathcal{U}_{\sigma}$. Then, we know that $\mathbf{u}_{\sigma} \in \mathcal{U}_{\sigma, a d}$ and the following estimate holds

$$
\left\|\mathbf{u}_{\sigma}-\overline{\mathbf{u}}\right\|_{\mathbf{H}^{1}\left(\Omega_{T}\right)^{*}} \leq C h\left\|\mathbf{u}_{\sigma}-\overline{\mathbf{u}}\right\|_{\mathbf{L}^{2}\left(\Omega_{T}\right)} .
$$

Now, from (4.22) we get

$$
\begin{aligned}
\frac{\kappa}{2}\left\|\mathbf{y}_{\overline{\mathbf{u}}_{\sigma}}-\overline{\mathbf{y}}\right\|_{\mathbf{L}^{2}\left(\Omega_{T}\right)}^{2} \leq & \left(J\left(\overline{\mathbf{u}}_{\sigma}\right)-J_{\sigma}\left(\overline{\mathbf{u}}_{\sigma}\right)\right)+\left(J_{\sigma}\left(\overline{\mathbf{u}}_{\sigma}\right)-J_{\sigma}\left(\mathbf{u}_{\sigma}\right)\right)+\left(J_{\sigma}\left(\mathbf{u}_{\sigma}\right)-J\left(\mathbf{u}_{\sigma}\right)\right) \\
& +\left(J\left(\mathbf{u}_{\sigma}\right)-J(\overline{\mathbf{u}})\right)=I_{1}+I_{2}+I_{3}+I_{4} .
\end{aligned}
$$

Let us study each term $I_{i}$. For the estimate of $I_{1}$ we use (4.1) and (4.12) as follows

$$
\begin{aligned}
& J\left(\overline{\mathbf{u}}_{\sigma}\right)-J_{\sigma}\left(\overline{\mathbf{u}}_{\sigma}\right) \leq \frac{1}{2} \int_{0}^{T} \int_{\Omega \backslash \Omega_{h}}\left|\mathbf{y}_{d}\right|^{2} \mathrm{~d} x \mathrm{~d} t+\frac{1}{2} \int_{0}^{T} \int_{\Omega_{h}}\left(\left|\mathbf{y}_{\overline{\mathbf{u}}_{\sigma}}-\mathbf{y}_{d}\right|^{2}-\left|\overline{\mathbf{y}}_{\sigma}-\mathbf{y}_{d}\right|^{2}\right) \mathrm{d} x \mathrm{~d} t \\
& \leq \frac{1}{2} \sqrt{T \mid \Omega \backslash \Omega_{h}} \mid\left\|\mathbf{y}_{d}\right\|_{\mathbf{L}^{4}\left(\Omega \backslash \Omega_{h}\right)}^{2}+\frac{1}{2}\left(\left\|\mathbf{y}_{\overline{\mathbf{u}}_{\sigma}}\right\|_{\mathbf{L}^{2}\left(\Omega_{T}\right)}+\left\|\overline{\mathbf{y}}_{\sigma}\right\|_{\mathbf{L}^{2}\left(\Omega_{T}\right)}\right)\left\|\mathbf{y}_{\overline{\mathbf{u}}_{\sigma}}-\overline{\mathbf{y}}_{\sigma}\right\|_{\mathbf{L}^{2}\left(\Omega_{T}\right)} \\
& \leq C\left(h\left\|\mathbf{y}_{d}\right\|_{\mathbf{L}^{4}\left(\Omega \backslash \Omega_{h}\right)}^{2}+h^{2}\right) .
\end{aligned}
$$

Let us observe that

$$
\lim _{|\sigma| \rightarrow 0}\left\|\mathbf{y}_{d}\right\|_{\mathbf{L}^{4}\left(\Omega \backslash \Omega_{h}\right)}^{2}=0
$$


To analyze $I_{2}$ we take $\sigma_{0}$ such that $\mathbf{u}_{\sigma} \in \bar{B}_{\varepsilon}(\overline{\mathbf{u}})$ for $|\sigma| \leq \sigma_{0}$, where $\bar{B}_{\varepsilon}(\overline{\mathbf{u}})$ is the ball given in the statement of the theorem. Since $\mathbf{u}_{\sigma} \in \mathcal{U}_{\sigma, a d} \cap \bar{B}_{\varepsilon}(\overline{\mathbf{u}})$, then $J_{\sigma}\left(\overline{\mathbf{u}}_{\sigma}\right) \leq J_{\sigma}\left(\mathbf{u}_{\sigma}\right)$, hence $I_{2} \leq 0$. $I_{3}$ is estimated in the same way as $I_{1}$, therefore the inequality (4.26) is also valid for $I_{3}$. To estimate $I_{4}$ we use the mean value theorem, the expression (3.1) for the derivative of $J$ and (4.24) to obtain

$$
\begin{aligned}
I_{4} & =J^{\prime}\left(\overline{\mathbf{u}}+\theta\left(\mathbf{u}_{\sigma}-\overline{\mathbf{u}}\right)\right)\left(\mathbf{u}_{\sigma}-\overline{\mathbf{u}}\right)=\int_{\Omega_{T}} \boldsymbol{\varphi}_{\theta}\left(\mathbf{u}_{\sigma}-\overline{\mathbf{u}}\right) \mathrm{d} x \mathrm{~d} t \\
& \leq\left\|\boldsymbol{\varphi}_{\theta}\right\|_{\mathbf{H}^{1}\left(\Omega_{T}\right)}\left\|\mathbf{u}_{\sigma}-\overline{\mathbf{u}}\right\|_{\mathbf{H}^{1}\left(\Omega_{T}\right)^{*}} \leq C h\left\|\mathbf{u}_{\sigma}-\overline{\mathbf{u}}\right\|_{\mathbf{L}^{2}\left(\Omega_{T}\right)},
\end{aligned}
$$

where $\boldsymbol{\varphi}_{\theta}$ denotes the adjoint state corresponding to $\overline{\mathbf{u}}+\theta\left(\mathbf{u}_{\sigma}-\overline{\mathbf{u}}\right)$. Collecting the estimates for $I_{i}, 1 \leq i \leq 4$, we deduce

$$
\lim _{|\sigma| \rightarrow 0} \frac{1}{|\sigma|}\left\|\mathbf{y}_{\overline{\mathbf{u}}_{\sigma}}-\overline{\mathbf{y}}\right\|_{\mathbf{L}^{2}\left(\Omega_{T}\right)}=0
$$

This inequality along with (4.23) imply (4.21).

Remark 4.8. If we follow the variational discretization approach to approximate (P) (see [19]), then $\mathcal{U}_{\sigma}=$ $\mathbf{L}^{\infty}\left(\Omega_{T}\right)$ and $\mathcal{U}_{\sigma, a d}=\mathcal{U}_{a d}$. Then, the Theorems 4.4 and 4.6 remain valid and the estimate (4.21) can be improved to get

$$
\left\|\overline{\mathbf{y}}_{\sigma}-\overline{\mathbf{y}}\right\|_{\mathbf{L}^{2}\left(\Omega_{T}\right)} \leq C h,
$$

assuming that $\mathbf{y}_{d} \in \mathbf{L}^{\infty}\left(\Omega_{T}\right)$. Indeed, first we replace the estimate (4.25) by

$$
\begin{aligned}
\frac{\kappa}{2}\left\|\mathbf{y}_{\overline{\mathbf{u}}_{\sigma}}-\overline{\mathbf{y}}\right\|_{\mathbf{L}^{2}\left(\Omega_{T}\right)}^{2} & \leq\left(J\left(\overline{\mathbf{u}}_{\sigma}\right)-J_{\sigma}\left(\overline{\mathbf{u}}_{\sigma}\right)\right) \\
& +\left(J_{\sigma}\left(\overline{\mathbf{u}}_{\sigma}\right)-J_{\sigma}(\overline{\mathbf{u}})\right)+\left(J_{\sigma}(\overline{\mathbf{u}})-J(\overline{\mathbf{u}})\right)=I_{1}+I_{2}+I_{3} .
\end{aligned}
$$

Now, the estimate for $I_{1}$ can be improved: $I_{1} \leq C h^{2}$. It is enough to argue as in the proof of Theorem 4.7 and to use the boundedness of $\mathbf{y}_{d}$ and (4.1)

$$
\int_{0}^{T} \int_{\Omega \backslash \Omega_{h}}\left|\mathbf{y}_{d}\right|^{2} \mathrm{~d} x \mathrm{~d} t \leq T\left|\Omega \backslash \Omega_{h}\right|\left\|\mathbf{y}_{d}\right\|_{\mathbf{L}^{\infty}\left(\Omega_{T}\right)}^{2} \leq C h^{2} .
$$

The same argument can be used to estimate $I_{3}$. For $I_{2}$ we recall that $\overline{\mathbf{u}} \in \mathcal{U}_{\sigma, a d} \cap \bar{B}_{\varepsilon}(\overline{\mathbf{u}})=\mathcal{U}_{a d} \cap \bar{B}_{\varepsilon}(\overline{\mathbf{u}})$, hence $I_{2} \leq 0$. Finally, these estimates and (4.23) lead to (4.27).

Remark 4.9. The numerical analysis of the velocity tracking problem for the $3 \mathrm{~d}$ Navier-Stokes system with bang-bang controls requires a special care. For instance one of the key ingredients of our proof, the improved error estimate in $L^{2}\left(0, T ; L^{2}(\Omega)\right)$ of Theorem 4.2, is proved in the two dimensional case and relies of embedding results that are valid only in $2 \mathrm{~d}$. The extension of this result in the three dimensional case, under our limited regularity assumptions, needs to be carefully examined.

\section{REFERENCES}

[1] F. Abergel and R. Temam, On some control problems in fluid mechanics. Theor. Comput. Fluid Dyn. 1 (1990) $303-325$.

$[2]$ E. Casas, An optimal control problem governed by the evolution Navier-Stokes equations, in Optimal Control of Viscous Flows, edited by S. Sritharan. Frontiers in Applied Mathematics. SIAM, Philadelphia, (1998).

[3] E. Casas, Second order analysis for bang-bang control problems of PDEs. SIAM J. Control Optim. 50 (2012) $2356-2372$.

[4] E. Casas and K. Chrysafinos, A discontinuous Galerkin time-stepping scheme for the velocity tracking problem. SIAM J. Numer. Anal. 50 (2012) 2281-2306.

[5] E. Casas and K. Chrysafinos, Error estimates for the discretization of the velocity tracking problem. Numer. Math. 130 (2015) 615-643.

[6] E. Casas, and K. Chrysafinos, Analysis of the velocity tracking control problem for the 3d evolutionary Navier-Stokes equations. SIAM J. Control Optim. 54 (2016) 99-128.

[7] E. Casas and F. Troltzsch, Second order analysis for optimal control problems: Improving results expected from from abstract theory. SIAM J. Optim. 22 (2012) 261-279. 
[8] E. Casas and F. Troltzsch, Second order optimality conditions and their role in pde control. Jahresbericht der Deutschen Mathematiker-Vereinigung 117 (2015) 3-44.

[9] E. Casas, M. Mateos and J.-P. Raymond, Error estimates for the numerical approximation of a distributed control problem for the steady-state navier-stokes equations. SIAM J. Control Optim. 46 (2007) 952-982.

[10] E. Casas, C. Ryll and F. Tröltzsch, Second Order and Stability Analysis for Optimal Sparse Control of the FitzHugh-Nagumo Equation. SIAM J. Control Optim. 53 (2015) 2168-2202.

[11] K. Chrysafinos, Convergence of discontinuous Galerkin approximations of an optimal control problem associated to semilinear parabolic pde's. ESAIM: M2AN 44 (2010) 189-206.

[12] K. Deckelnick and M. Hinze, Semidiscretization and error estimates for distributed control of the instationary Navier-Stokes equations. Numer. Math. 97 (2004) 297-320.

[13] J. Douglas Jr., T. Dupont and L. Wahlbin, The stability in $L^{q}$ of the $L^{2}$ projection into finite element function spaces. Numer. Math. 23 (1975) 193-197.

[14] J.C. Dunn, On second order sufficient optimality conditions for structured nonlinear programs in infinite-dimensional function spaces. In Mathematical Programming with Data Perturbations, edited by A. Fiacco. Marcel Dekker (1998) 83-107.

[15] P. Girault and P. Raviart, Finite Element Methods for Navier-Stokes Equations. Theory and Algorithms. Springer-Verlag, Berlin, Heidelberg, New-York, Tokyo (1986).

[16] M.D. Gunzburger, Perspectives in flow control and optimization. Advances in Design and Control SIAM, Philadelphia (2003).

[17] M.D. Gunzburger and S. Manservisi, The velocity tracking problem for navier-stokes flows with bounded distributed control. SIAM J. Control Optim. 37 (1999) 1913-1945.

[18] M.D. Gunzburger and S. Manservisi, Analysis and approximation of the velocity tracking problem for Navier-Stokes flows with distributed control. SIAM J. Numer. Anal. 37 (2000) 1481-1512.

[19] M. Hinze, A Variational discretization concept in control constrained optimization: The linear quadratic case. Comput. Optim. Appl. 30 (2005) 45-61.

[20] M. Hinze and K. Kunisch, Second order methods for optimal control of time-dependent fluid flow. SIAM J. Control Optim. 40 (2001) 925-946.

[21] O. Ladyzhenskaya, The Mathematical Theory of Viscous Incompressible Flow. English translation, second edition. Gordon and Breach, New York (1969).

[22] O. Ladyzhenskaya, V.A. Solonnikov and N.N. Ural'tseva, Linear and Quasilinear Equations of Parabolic Type. American Mathematical Society (1988).

[23] J.-L. Lions, Quelques Méthodes de Résolution des Problèmes aux Limites non Linéaires. Dunod, Paris (1969).

[24] D. Meidner and B. Vexler, A priori error estimates for the space-time finite element discretization of parabolic optimal control problems. Part I: Problems without control constraints. SIAM J. Control Optim. 47 (2008) 1150-1177.

[25] D. Meidner and B. Vexler, A priori error estimates for the space-time finite element discretization of parabolic optimal control problems. Part II: Problems with control constraints. SIAM J. Control Optim. 47 (2008) 1301-1329.

[26] I. Neitzel and B. Vexler, A priori error estimates for space-time finite element discretization of semilinear parabolic optimal control problems. Numer. Math. 120 (2012) 345-386.

[27] P.A Raviart and J.M. Thomas, Introduction à L'analyse Numérique des Equations aux Dérivées Partielles. Masson, Paris (1983).

[28] A. Rösch, Error estimates for parabolic optimal control problems with control constraints. Z. Anal. Anwendungen 23 (2004) 353-376

[29] V. Solonnikov, Estimates for solutions of nonstationary Navier-Stokes equaions. J. Soviet. Math. 8 (1977) $213-317$.

[30] S.S. Sritharan, Optimal control of viscous flow. SIAM, Philadelphia (1998).

[31] R. Temam, Navier-Stokes Equations. North-Holland, Amsterdam (1979).

[32] F. Tröltzsch and D. Wachsmuth, Second-order suficcient optimality conditions for the optimal control of Navier-Stokes equations. ESAIM: COCV 12 (2006) 93-119. 